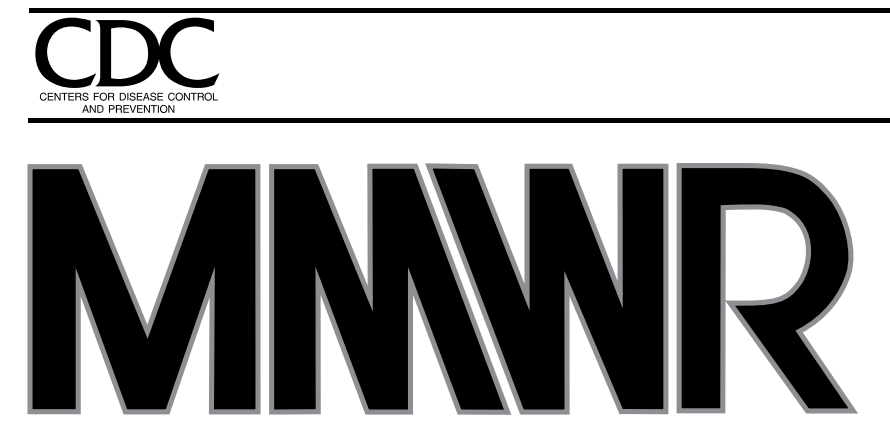

December 23, 1994 / Vol. 43 / No. 50

925 Cigarette Smoking Among Adults United States, 1993

930 Abortion Surveillance: Preliminary Data - United States, 1992

939 State-Specific Trends Among Women Who Did Not Receive Prenatal Care United States, 1980-1992

943 Bolivian Hemorrhagic Fever El Beni Department, Bolivia, 1994

MORBIDITY AND MORTALITY WEEKLY REPORT

946 Notices to Readers

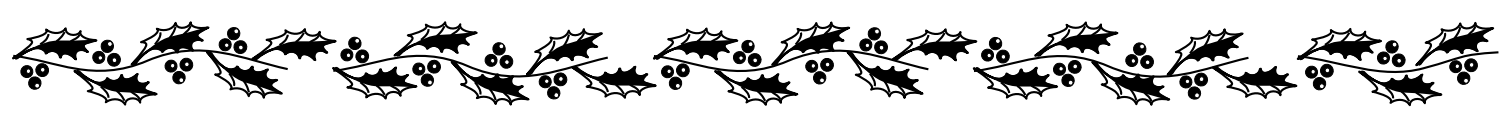

Health Objectives for the Nation

\title{
Cigarette Smoking Among Adults — United States, 1993
}

The annual prevalence of cigarette smoking among adults in the United States declined $40 \%$ during $1965-1990$ (from $42.4 \%$ to $25.5 \%$ ) (1) but was virtually unchanged during 1990-1992 (2). To determine the prevalence of smoking among adults, smoker interest in quitting, and the prevalence of cessation (i.e., quit ratio) among adults during 1993, the Year 2000 Health Objectives Supplement of the 1993 National Health Interview Survey (NHIS-2000) collected self-reported information about cigarette smoking from a random sample of civilian, noninstitutionalized adults aged $\geq 18$ years. This report presents the prevalence estimates for 1993 and compares them with estimates from the 1992 Cancer Epidemiology Supplement and presents 1993 estimates for smoker interest in quitting completely and the prevalence of cessation among ever smokers.

The overall response rate for the 1993 NHIS-2000 $(n=20,860)$ was $81.2 \%$. For 1993, current smoking status was determined through two questions: "Have you smoked at least 100 cigarettes in your entire life?" and "Do you now smoke cigarettes every day, some days, or not at all?" Ever smokers were persons who reported having smoked at least 100 cigarettes during their entire lives. Current smokers were defined as those who had smoked 100 cigarettes and now smoked either every day (i.e., daily smokers) or some days (i.e., some-day smokers). Former smokers had smoked at least 100 cigarettes in their lives but did not currently smoke. The prevalence of cessation was the percentage of former smokers among ever smokers. Interest in quitting smoking was assessed using answers to the question "Would you like to completely stop smoking cigarettes?" Data were adjusted for nonresponse and weighted to provide national estimates. Confidence intervals $(\mathrm{Cls})$ were calculated using standard errors generated by the Software for Survey Data Analysis (SUDAAN) (3).

Prevalence estimates for 1992 were based on two definitions of current smoking and were calculated by averaging the estimates generated by each definition ( 2 ). One of the 1992 definitions of current smoking (smoking every day or some days) was identical to the definition used in 1993; these estimates are compared in this report.

In 1993 , an estimated 46 million $(25.0 \%[95 \% \mathrm{Cl}= \pm 0.7 \%])$ adults in the United States were current smokers (Table 1): $20.4 \%(95 \% \mathrm{Cl}= \pm 0.7 \%)$ were daily smokers, and $4.6 \%$

U.S. DEPARTMENT OF HEALTH AND HUMAN SERVICES / Public Health Service 
TABLE 1. Percentage of adults aged $\geq 18$ years who were current cigarette smokers, ${ }^{*}$ by sex, race/ethnicity, education level, age group, and socioeconomic status - National Health Interview Survey (NHIS), United States, 1992 and $1993^{\dagger}$

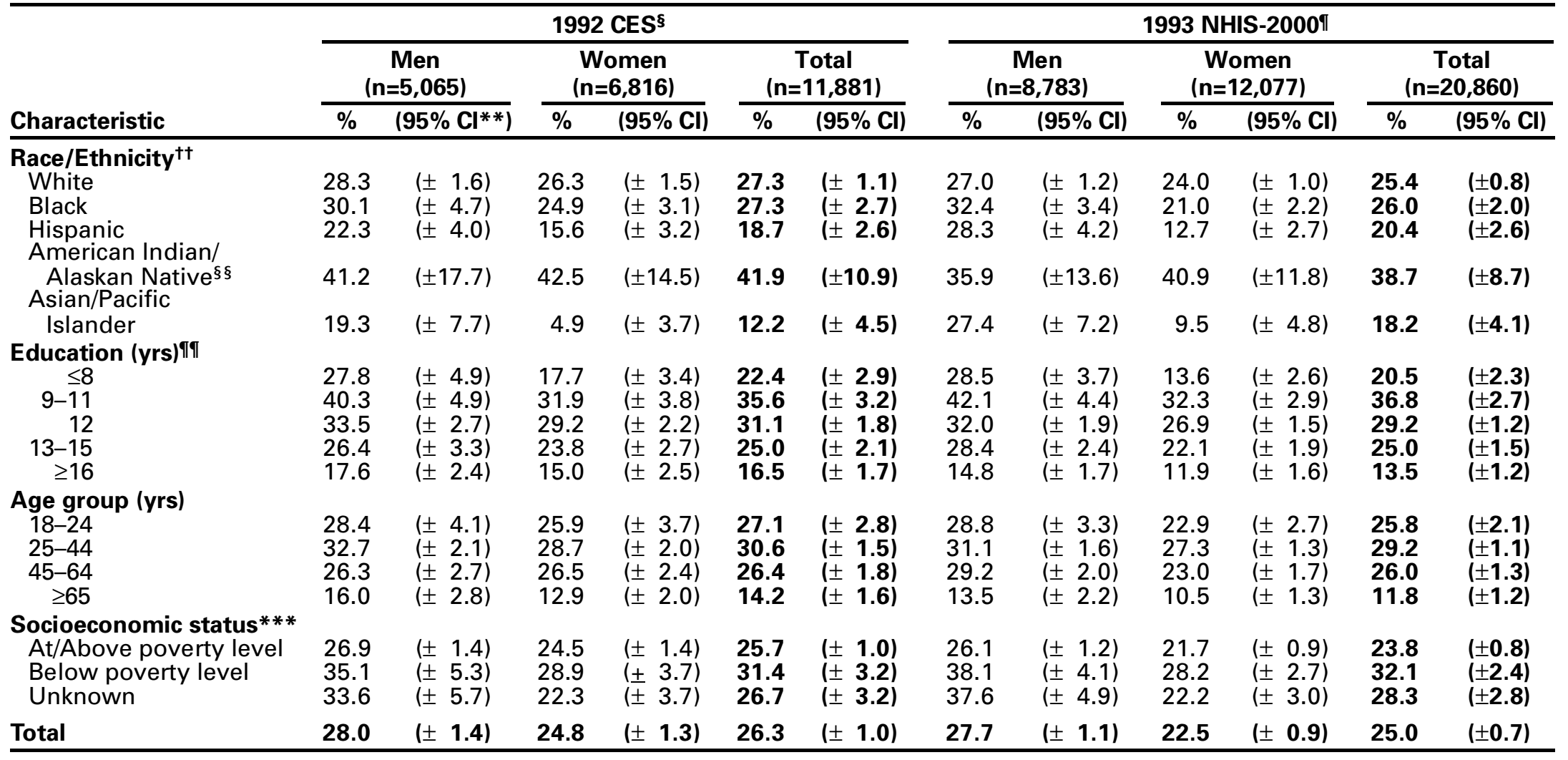

* Persons who reported having smoked at least 100 cigarettes and who reported now smoking every day or some days.

† Excludes 168 respondents with unknown smoking status.

§ Year 2000 Health Objectives Supplement.

* Confidence interval.

t† Excludes 257 respondents in unknown, multiple, and other race categories.

$\S \S$ Estimates should be interpreted with caution because of the small number of cases.

IT Persons aged $\geq 25$ years.

** Poverty statistics are based on definitions developed by the Social Security Administration in 1964, subsequently modified by federal interagency committees in 1969 and 1980, and prescribed by the Office of Management and Budget as the standard to be used by federal agencies for statistical purposes. 
Cigarette Smoking - Continued

(95\% $\mathrm{Cl}= \pm 0.3 \%$ ) were some-day smokers. Smoking prevalence was significantly higher among men $(27.7 \%$ [95\% $\mathrm{Cl}= \pm 1.1 \%$ ] [24 million men]) than among women (22.5\% [95\% Cl= $\pm 0.9 \%$ ] [22 million women]) (Table 1). The racial/ethnic group-specific prevalence was highest among American Indians/Alaskan Natives $138.7 \%$ [95\%

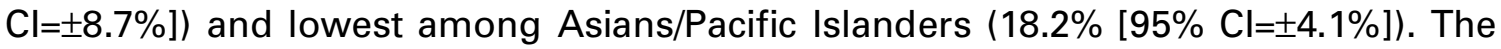
prevalence of smoking among persons with $\leq 8$ years of education was significantly lower than that among persons with 9-15 years of education; however, among persons with $\geq 9$ years of education, prevalences varied inversely with education level. For all groups, the prevalence of smoking was highest among males who had dropped out of high school $(42.1 \%$ [95\% $\mathrm{Cl}= \pm 4.4 \%])$. Smoking prevalence was higher among persons living below the poverty level* $(32.1 \%$ [95\% $\mathrm{Cl}= \pm 2.4 \%])$ than among those living at or above the poverty level $(23.8 \%[95 \% \mathrm{Cl}= \pm 0.8 \%])$.

The prevalence of current smokers in 1993 was unchanged statistically from 1992 (25.0\% and $26.3 \%$, respectively). However, the prevalence of daily smoking in 1993 (20.4\% [95\% Cl= $=0.7 \%]$ ) was significantly lower than in $1992(22.3 \%[95 \% \mathrm{Cl}= \pm 0.9 \%])$. In addition, prevalence estimates for current smokers during 1993 were lower overall for women, persons with a college education or higher, total persons living at or above the poverty level, and women living at or above the poverty level (Table 1).

Of current smokers, an estimated 32 million persons $(69.7 \%$ [95\% $\mathrm{Cl}= \pm 1.6 \%])$ reported they wanted to quit smoking completely. Women were more likely to report an

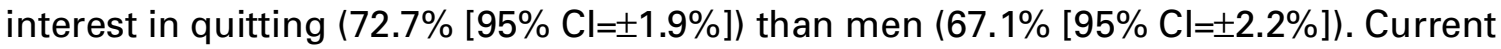
smokers aged $\geq 65$ years $(49.9 \%$ [ $95 \% \mathrm{Cl}=5.8 \%]$ ) were the least likely to report that they wanted to completely stop smoking.

In 1993, an estimated 46 million adults were former smokers $149.6 \%$ [95\% $\mathrm{Cl}= \pm 1.2 \%$ ] of ever smokers) (Table 2 ). The prevalence of cessation was higher among men $(51.9 \%$ [95\% $\mathrm{Cl}= \pm 1.5 \%])$, whites $(51.6 \%$ [95\% $\mathrm{Cl}= \pm 1.3 \%]$ ), and persons living at or above the poverty level $(52.4 \%[95 \% \mathrm{Cl}= \pm 1.2 \%])$, and increased directly with age. Among education levels, the prevalence of cessation was lowest among persons with 9-11 years of education (38.2\% [95\% $\mathrm{Cl}= \pm 3.3 \%]$ ).

Reported by: Epidemiology Br, Office on Smoking and Health, National Center for Chronic Disease Prevention and Health Promotion, CDC.

Editorial Note: Although the overall prevalence of current smoking did not change from 1992 to 1993 , the prevalence of daily smoking declined during 1993, possibly reflecting the proliferation of restrictive worksite and public smoking policies (4). In addition, the relatively greater decline among women is consistent with a previous report that, in workplace settings, women may be more likely to quit smoking because of worksite smoking bans (5).

Differences in prevalence among racial/ethnic groups may be influenced by differences in education levels and socioeconomic status, as well as by social and cultural phenomena. For example, in a recent report $(6)$, the prevalence of behavioral risk factors, including cigarette smoking, was generally higher among persons with $\leq 12$ years of education.

\footnotetext{
* Poverty statistics are based on a definition originated by the Social Security Administration in 1964, subsequently modified by federal interagency committees in 1969 and 1980, and prescribed by the Office of Management and Budget as the standard to be used by federal agencies for statistical purposes.
} 
Cigarette Smoking - Continued

TABLE 2. Percentage of interest in quitting among current smokers aged $\geq 18$ years* and prevalence of cessation among ever smokers aged $\geq 18$ years, ${ }^{\dagger}$ by sex, race/ethnicity, education level, age group, and socioeconomic status - National Health Interview Survey, United States, $1993^{\S}$

\begin{tabular}{|c|c|c|c|c|}
\hline \multirow[b]{2}{*}{ Characteristic } & \multicolumn{2}{|c|}{$\begin{array}{c}\text { Interest in quitting } \\
\text { among current smokers } \\
(n=5,261)\end{array}$} & \multicolumn{2}{|c|}{$\begin{array}{c}\text { Prevalence of cessation } \\
\text { among ever smokers } \\
(n=10,370)\end{array}$} \\
\hline & $\%$ & (95\% ClT) & $\%$ & $(95 \% \mathrm{Cl})$ \\
\hline $\begin{array}{l}\text { Sex } \\
\text { Men } \\
\text { Women }\end{array}$ & $\begin{array}{l}67.1 \\
72.7\end{array}$ & $\begin{array}{l}( \pm 2.2) \\
( \pm 1.9)\end{array}$ & $\begin{array}{l}51.9 \\
46.7\end{array}$ & $\begin{array}{l}( \pm 1.5) \\
( \pm 1.6)\end{array}$ \\
\hline $\begin{array}{l}\text { Race/Ethnicity** } \\
\text { White } \\
\text { Black } \\
\text { Hispanic } \\
\text { American Indian/ } \\
\text { Alaskan Native }{ }^{\dagger \dagger} \\
\text { Asian/Pacific Islander }\end{array}$ & $\begin{array}{l}70.0 \\
71.4 \\
68.7 \\
65.0 \\
60.2\end{array}$ & $\begin{array}{l}( \pm 1.8) \\
( \pm 4.8) \\
( \pm 5.8) \\
( \pm 14.5) \\
( \pm 12.2)\end{array}$ & $\begin{array}{l}51.6 \\
37.8 \\
44.3 \\
\\
35.1 \\
46.1\end{array}$ & $\begin{array}{l}( \pm 1.3) \\
( \pm 3.4) \\
( \pm 5.0) \\
( \pm 16.6) \\
( \pm 8.7)\end{array}$ \\
\hline $\begin{array}{c}\text { Education }(\mathbf{y r s})^{\S \S} \\
\leq 8 \\
9-11 \\
12 \\
13-15 \\
\geq 16\end{array}$ & $\begin{array}{l}62.6 \\
67.8 \\
71.5 \\
71.8 \\
67.5\end{array}$ & $\begin{array}{l}( \pm 5.5) \\
( \pm 4.4) \\
( \pm 2.2) \\
( \pm 3.6) \\
( \pm 4.5)\end{array}$ & $\begin{array}{l}56.2 \\
38.2 \\
45.3 \\
50.7 \\
65.4\end{array}$ & $\begin{array}{l}( \pm 3.9) \\
( \pm 3.3) \\
( \pm 1.7) \\
( \pm 2.3) \\
( \pm 2.5)\end{array}$ \\
\hline $\begin{array}{l}\text { Age group (yrs) } \\
18-24 \\
25-44 \\
45-64 \\
\geq 65\end{array}$ & $\begin{array}{l}68.6 \\
73.7 \\
68.5 \\
49.9\end{array}$ & $\begin{array}{l}( \pm 4.5) \\
( \pm 2.0) \\
( \pm 3.0) \\
( \pm 5.8)\end{array}$ & $\begin{array}{l}21.7 \\
39.0 \\
56.6 \\
76.6\end{array}$ & $\begin{array}{l}( \pm 3.1) \\
( \pm 1.5) \\
( \pm 2.0) \\
( \pm 2.1)\end{array}$ \\
\hline $\begin{array}{l}\text { Socioeconomic status } 19 \\
\text { At/Above poverty level } \\
\text { Below poverty level } \\
\text { Unknown }\end{array}$ & $\begin{array}{l}70.7 \\
69.7 \\
59.0\end{array}$ & $\begin{array}{l}( \pm 1.8) \\
( \pm 3.8) \\
( \pm 5.6)\end{array}$ & $\begin{array}{l}52.4 \\
30.4 \\
41.6\end{array}$ & $\begin{array}{l}( \pm 1.2) \\
( \pm 3.1) \\
( \pm 4.3)\end{array}$ \\
\hline$\underline{\text { Total }}$ & 69.7 & $( \pm 1.6)$ & 49.6 & $( \pm 1.2)$ \\
\hline
\end{tabular}

* Persons who reported having smoked at least 100 cigarettes and who reported now smoking every day or some days.

${ }^{\dagger}$ Persons who reported ever smoking 100 cigarettes during their lifetime.

${ }^{\S}$ Excludes 168 respondents with unknown smoking status.

IConfidence interval.

**Excludes 257 respondents in unknown, multiple, and other race categories.

${ }^{\dagger \dagger}$ Estimates should be interpreted with caution because of the small number of cases.

$\S \S$ Persons aged $\geq 25$ years.

TI Poverty statistics are based on definitions developed by the Social Security Administration in 1964, subsequently modified by federal interagency committees in 1969 and 1980, and prescribed by the Office of Management and Budget as the standard to be used by federal agencies for statistical purposes.

From 1992 to 1993 , daily smoking prevalence increased among high school seniors from $17.2 \%$ to $19.0 \%$ (1). To be effective, school-based prevention programs should begin in kindergarten and continue through high school. This intervention should be especially intensive in middle school and should be reinforced in high school. CDC has published guidelines for incorporating tobacco-use prevention and cessation strate- 


\section{Cigarette Smoking - Continued}

gies in the early grades in schools (7). School-based programs should provide instruction about the short- and long-term physiologic and social consequences of tobacco use, social influences on tobacco use, peer norms regarding tobacco use, and refusal skills.

The findings in this report are subject to at least two limitations. First, because the 1992 and 1993 estimates are based on data collected during a 6-month period, these estimates may not be representative of annual prevalence. In particular, other data suggest that the restriction of the surveys to these periods may have minimized the true magnitude of declines in prevalence (National Household Survey on Drug Abuse, unpublished data, 1992 and 1993). Second, because these estimates are based on selfreported data, prevalences may be underestimated. However, underreporting is believed to be low in national prevalence surveys $(8)$.

To sustain the decline in smoking prevalence, efforts must be intensified to discourage initiation and to promote cessation. Although $70 \%$ of smokers want to stop smoking and $34 \%$ attempt to quit each year, only $2.5 \%$ successfully stop smoking each year (9). The high rate of relapse is a consequence of the effect of nicotine dependence. Smokers who need assistance with stopping can receive self-help materials from local voluntary agencies, CDC (telephone [800] 232-1311 or [404] 488-5705), and the National Institutes of Health (telephone [800] 422-6237). Many smokers are addicted to nicotine and could potentially benefit from nicotine replacement therapy (NRT); NRT and other cessation assistance can be obtained from physicians and dentists. Information about formal cessation programs can be obtained from local voluntary agencies or health-care providers.

The health risks of cigarette smoking can be eliminated only by quitting; switching to lower "tar" and nicotine cigarettes is not a safe alternative (10). Comprehensive measures for promoting cessation and reducing the prevalence of smoking include increasing tobacco excise taxes, enforcing minors' access laws, restricting smoking in public places, restricting tobacco advertising and promotion, and conducting counteradvertising campaigns.

\section{References}

1. Giovino GA, Schooley MW, Zhu B-P, et al. Surveillance for selected tobacco-use behaviorsUnited States, 1900-1994. MMWR 1994;43(no. SS-3).

2. CDC. Cigarette smoking among adults-United States, 1992, and changes in the definition of current cigarette smoking. MMWR 1994;43:342-6.

3. Shah BV. Software for Survey Data Analysis (SUDAAN), version 5.50 [Software documentation]. Research Triangle Park, North Carolina: Research Triangle Institute, 1991.

4. Evans NJ, Gilpin E, Pierce JP, et al. Occasional smoking among adults: evidence from the California Tobacco Survey. Tobacco Control 1992;1:169-75.

5. Brenner $\mathrm{H}$, Mielck A. Smoking prohibition in the workplace and smoking cessation in the Federal Republic of Germany. Prev Med 1992;21:252-61.

6. CDC. Prevalence of selected risk factors for chronic disease by education level in racial/ethnic populations-United States, 1991-1992. MMWR 1994;43:894-9.

7. CDC. Guidelines for school health programs to prevent tobacco use and addiction. MMWR 1994;43(no. RR-2).

8. CDC. The health benefits of smoking cessation: a report of the Surgeon General, 1990. Rockville, Maryland: US Department of Health and Human Services, Public Health Service, 1990; DHHS publication no. (CDC) $90-8416$.

9. CDC. Smoking cessation during previous year among adults-United States, 1990 and 1991. MMWR 1993;42:504-7. 
Cigarette Smoking - Continued

10. US Department of Health and Human Services. The health consequences of smoking: the changing cigarette-a report of the Surgeon General, 1981. Rockville, Maryland: US Department of Health and Human Services, Public Health Service, 1981; DHHS publication no. (PHS)81-50156.

\section{Current Trends}

\section{Abortion Surveillance: Preliminary Data — United States, 1992}

For 1992, CDC received data about legal induced abortions from 52 reporting areas (the 50 states, New York City, and the District of Columbia). This report presents preliminary data for 1992.

In 1992, a total of 1,359,145 legal abortions were reported to CDC (Table 1), a decrease of $2.1 \%$ from the number reported for 1991 (1), and the number of live births decreased by $1.1 \%$ (2). As a result, the national abortion ratio (number of legal abortions per 1000 live births) decreased from 339 in 1991 to 335 in 1992 (Figure 1). The national abortion rate (number of legal abortions per 1000 women aged 15-44 years) also declined from 24 in 1991 to 23 in 1992. This rate increased each year from 1972 to 1980 (when it peaked [25]); since 1980, the rate has remained stable, fluctuating from 23 to 24 . As in previous years, approximately $92 \%$ of women who had a legal abortion were residents of the state in which the procedure was performed.

Women who obtained legal abortions in 1992 were predominately aged $<25$ years, white, and unmarried. Compared with 1991, a lower proportion of women who had abortions in 1992 had had live-born children (48\% and 46\%, respectively). Curettage (suction and sharp) remained the primary abortion procedure (approximately $99 \%$ of all such procedures). As in previous years, approximately half of legal abortions were performed during the first 8 weeks of gestation (Table 1): $14 \%$ of abortions were performed at $\leq 6$ weeks, $15 \%$ at 7 weeks, and $21 \%$ at 8 weeks' gestation. As in previous years, approximately $89 \%$ of abortions were performed during the first 12 weeks of pregnancy.

Reported by: Statistics and Computer Resources Br, Div of Reproductive Health, National Center for Chronic Disease Prevention and Health Promotion, CDC.

Editorial Note: The annual number of abortions in the United States has remained relatively stable since 1980, varying each year by $\leq 5 \%$. However, since 1990 (the year in which the number of abortions was highest), the number of abortions has decreased each year. In 1992, the national ratio of abortions to live births was lower than for any year since 1977, indicating that a greater proportion of pregnancies ended in a live birth (3). The national fertility rate (number of live births per 1000 women of reproductive age [15-44 years]) also peaked in 1990 and has declined somewhat since then (2).

As in previous years, most women who obtained an abortion were white. However, the abortion rate for black women is approximately three times that for white women (CDC, unpublished data, 1991). Differences in abortion by race may reflect differences in socioeconomic status, education level, contraceptive use, and access to family planning, contraceptive, and abortion services. 


\section{Abortion Surveillance - Continued}

FIGURE 1. Fertility rate* and abortion ratio $^{\dagger}$ and rate, ${ }^{\S}$ by year - United States, 1972-1992

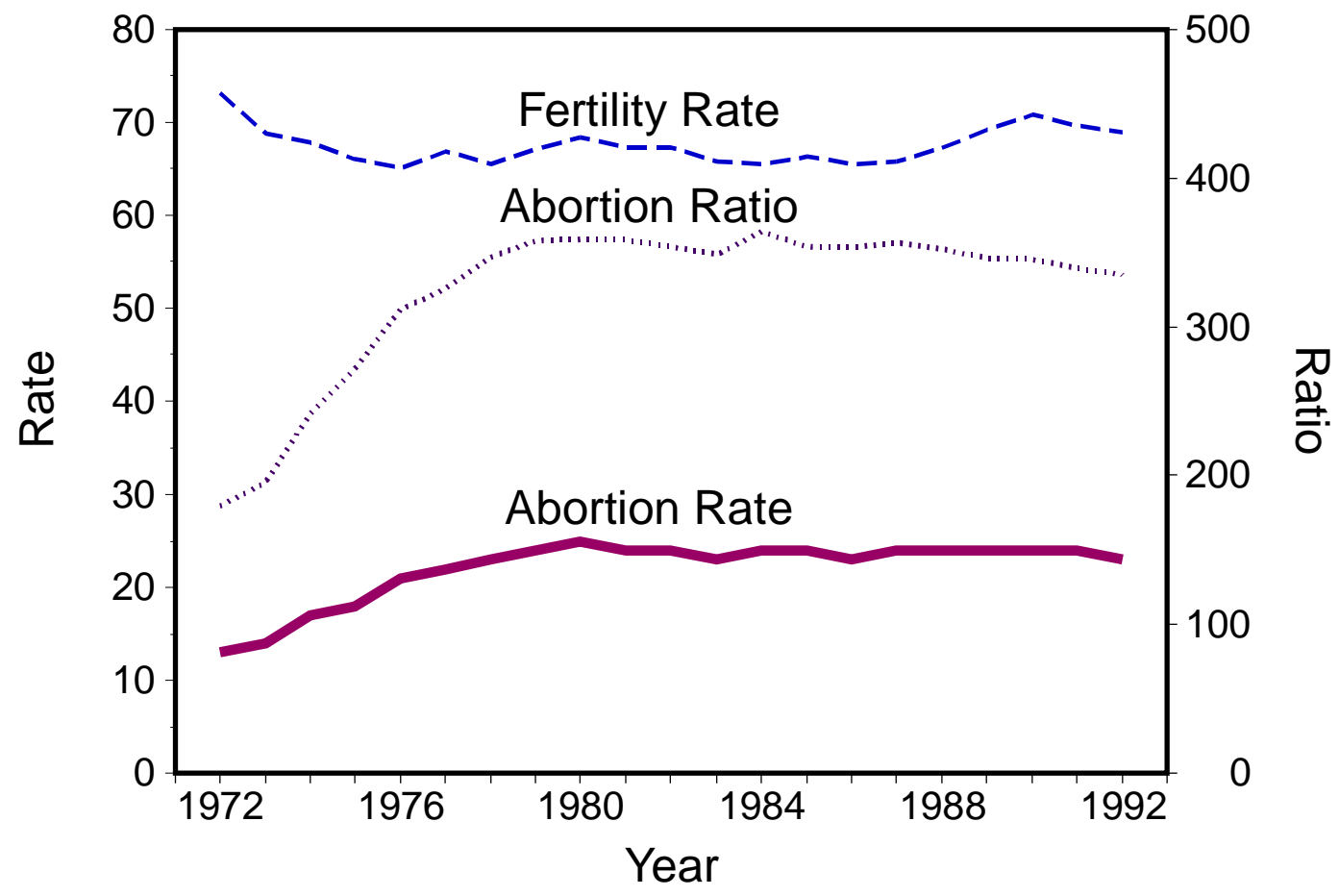

* Live births per 1000 women aged $15-44$ years.

t Number of legal induced abortions per 1000 live births.

$\S^{\S}$ Number of legal induced abortions per 1000 women aged 15-44 years.

Although the total number of legal induced abortions during 1992 was available for all 52 reporting areas, approximately $26 \%$ of the abortions were reported from states that do not have centralized reporting; these areas could not provide information on the characteristics of women obtaining abortions. Interpretation of temporal comparisons is constrained because the number of states that report characteristics varies each year.

Many states emphasize the prevention of unintended pregnancy, particularly among teenagers. Abortion and birth statistics both are essential to provide estimates of pregnancy rates. To assist efforts to prevent unintended pregnancy, an accurate assessment of abortion (including the number and characteristics of women obtaining legal abortions in all states) is needed on an ongoing basis. In 1992, most areas reported abortions at $\leq 8$ weeks of gestation by week of gestation for the first time. This approach to reporting will assist in monitoring trends in legal abortions.

Additional statistical and epidemiologic information about legal induced abortions is available from CDC's automated Reproductive Health Information line at (404) 330 1230, which provides information by fax, voice recordings, or mail. 
TABLE 1. Reported number of legal induced abortions, abortion ratios, ${ }^{*}$ abortion rates, ${ }^{\dagger}$ and characteristics of women who obtained legal induced abortions - United States, selected years, 1972-1992

\begin{tabular}{|c|c|c|c|c|c|c|c|c|c|}
\hline \multirow[b]{2}{*}{ Characteristic } & \multicolumn{9}{|c|}{ Year } \\
\hline & 1972 & 1976 & 1980 & 1985 & 1988 & 1989 & 1990 & 1991 & $1992^{\S}$ \\
\hline \multirow[t]{2}{*}{$\begin{array}{l}\text { Reported no. } \\
\text { legal induced } \\
\text { abortions } \\
\text { Abortion ratios } \\
\text { Abortion rates }\end{array}$} & $\begin{array}{c}586,760 \\
180 \\
13\end{array}$ & $\begin{array}{c}988,267 \\
312 \\
21\end{array}$ & $\begin{array}{c}1,297,606 \\
359 \\
25\end{array}$ & $\begin{array}{c}1,328,570 \\
354 \\
24\end{array}$ & $\begin{array}{c}1,371,285 \\
352 \\
24\end{array}$ & $\begin{array}{c}1,396,658 \\
346 \\
24\end{array}$ & $\begin{array}{c}1,429,577 \\
345 \\
24\end{array}$ & $\begin{array}{c}1,388,937 \\
339 \\
24\end{array}$ & $\begin{array}{c}1,359,145 \\
335 \\
23\end{array}$ \\
\hline & \multicolumn{9}{|c|}{ Percentage distribution? } \\
\hline \multicolumn{10}{|l|}{ Residence } \\
\hline $\begin{array}{l}\text { In-state } \\
\text { Out-of-state }\end{array}$ & $\begin{array}{l}56.2 \\
43.8\end{array}$ & $\begin{array}{l}90.0 \\
10.0\end{array}$ & $\begin{array}{r}92.6 \\
7.4\end{array}$ & $\begin{array}{r}92.4 \\
7.6\end{array}$ & $\begin{array}{r}91.4 \\
8.6\end{array}$ & $\begin{array}{r}91.0 \\
9.0\end{array}$ & $\begin{array}{r}91.8 \\
8.2\end{array}$ & $\begin{array}{r}91.6 \\
8.4\end{array}$ & $\begin{array}{r}91.7 \\
8.3\end{array}$ \\
\hline \multicolumn{10}{|l|}{ Age group (yrs) } \\
\hline $\begin{array}{r}\leq 19 \\
20-24\end{array}$ & $\begin{array}{l}32.6 \\
32.5\end{array}$ & $\begin{array}{l}32.1 \\
33.3\end{array}$ & $\begin{array}{l}29.2 \\
35.5\end{array}$ & $\begin{array}{l}26.3 \\
34.7\end{array}$ & $\begin{array}{l}25.3 \\
32.8\end{array}$ & $\begin{array}{l}24.2 \\
32.6\end{array}$ & $\begin{array}{l}22.4 \\
33.2\end{array}$ & $\begin{array}{l}21.0 \\
34.4\end{array}$ & $\begin{array}{l}20.1 \\
34.6\end{array}$ \\
\hline$\geq 25$ & 34.9 & 34.6 & 35.3 & 39.0 & 41.9 & 43.2 & 44.4 & 44.6 & 45.3 \\
\hline \multicolumn{10}{|l|}{ Race } \\
\hline $\begin{array}{l}\text { White } \\
\text { Black } \\
\text { Other }\end{array}$ & $\begin{array}{l}77.0 \\
23.0 \\
-\end{array}$ & $\begin{array}{c}66.6 \\
33.4 \\
-\end{array}$ & $\begin{array}{c}69.9 \\
30.1 \\
-\end{array}$ & $\begin{array}{r}66.6 \\
29.8 \\
3.5\end{array}$ & $\begin{array}{r}64.4 \\
31.1 \\
4.5\end{array}$ & $\begin{array}{r}64.2 \\
31.2 \\
4.6\end{array}$ & $\begin{array}{r}64.8 \\
31.8 \\
3.4\end{array}$ & $\begin{array}{r}63.8 \\
32.5 \\
3.7\end{array}$ & $\begin{array}{r}62.9 \\
33.3 \\
3.8\end{array}$ \\
\hline \multicolumn{10}{|l|}{ Ethnicity } \\
\hline Hispanic & - & - & - & - & - & - & 9.8 & 13.5 & 15.1 \\
\hline Non-Hispanic & - & - & - & - & - & - & 90.2 & 86.5 & 84.9 \\
\hline \multicolumn{10}{|l|}{ Marital status } \\
\hline $\begin{array}{l}\text { Married } \\
\text { Unmarried }\end{array}$ & $\begin{array}{l}29.7 \\
70.3\end{array}$ & $\begin{array}{l}24.6 \\
75.4\end{array}$ & $\begin{array}{l}23.1 \\
76.9\end{array}$ & $\begin{array}{l}19.3 \\
80.7\end{array}$ & $\begin{array}{l}20.3 \\
79.7\end{array}$ & $\begin{array}{l}20.1 \\
79.9\end{array}$ & $\begin{array}{l}21.7 \\
78.3\end{array}$ & $\begin{array}{l}21.4 \\
78.6\end{array}$ & $\begin{array}{l}20.7 \\
79.3\end{array}$ \\
\hline \multicolumn{10}{|l|}{ No. live births** } \\
\hline $\begin{array}{r}0 \\
1 \\
2 \\
3 \\
>4\end{array}$ & $\begin{array}{r}49.4 \\
18.2 \\
13.3 \\
8.7\end{array}$ & $\begin{array}{r}47.7 \\
20.7 \\
15.4 \\
8.3 \\
7.9\end{array}$ & $\begin{array}{r}58.4 \\
19.4 \\
13.7 \\
5.3 \\
3.2\end{array}$ & $\begin{array}{r}56.3 \\
21.6 \\
14.5 \\
5.1 \\
2.5\end{array}$ & $\begin{array}{r}52.4 \\
23.4 \\
16.0 \\
5.6 \\
2.6\end{array}$ & $\begin{array}{r}52.2 \\
23.6 \\
15.9 \\
5.7 \\
2.6\end{array}$ & $\begin{array}{r}49.2 \\
24.4 \\
16.9 \\
6.1 \\
3.4\end{array}$ & $\begin{array}{r}47.7 \\
25.1 \\
17.4 \\
6.4 \\
3.4\end{array}$ & $\begin{array}{r}46.3 \\
25.7 \\
17.9 \\
6.6 \\
3.5\end{array}$ \\
\hline
\end{tabular}




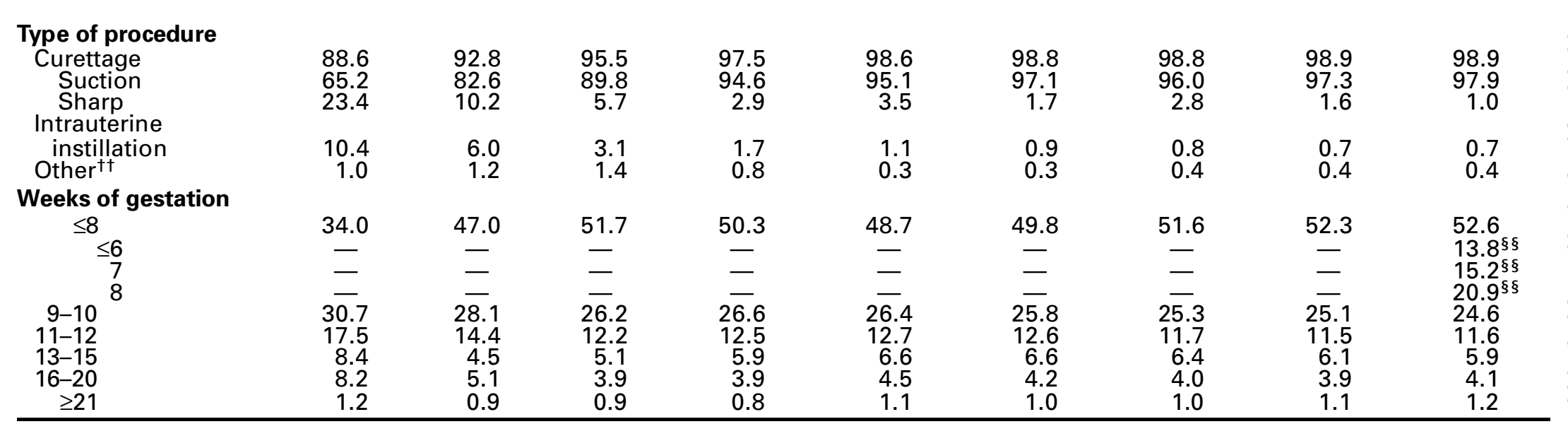

* Per 1000 live births.

tPer 1000 women aged $15-44$ years.

\$Preliminary data.

TExcludes unknown values. The number of areas reporting a given characteristic varied. For 1992, the number of areas reporting residence was 41; age, 42; race, 35; ethnicity, 21; marital status, 38; number of live births, 38; type of procedure, 38; and weeks of gestation, 38 .

* For 1972-1976, data indicate number of living children.

${ }^{t \dagger}$ Includes hysterotomy and hysterectomy.

$\S \S$ Data are for 34 reporting areas only. 
FIGURE I. Notifiable disease reports, comparison of 4-week totals ending December 17, 1994, with historical data - United States

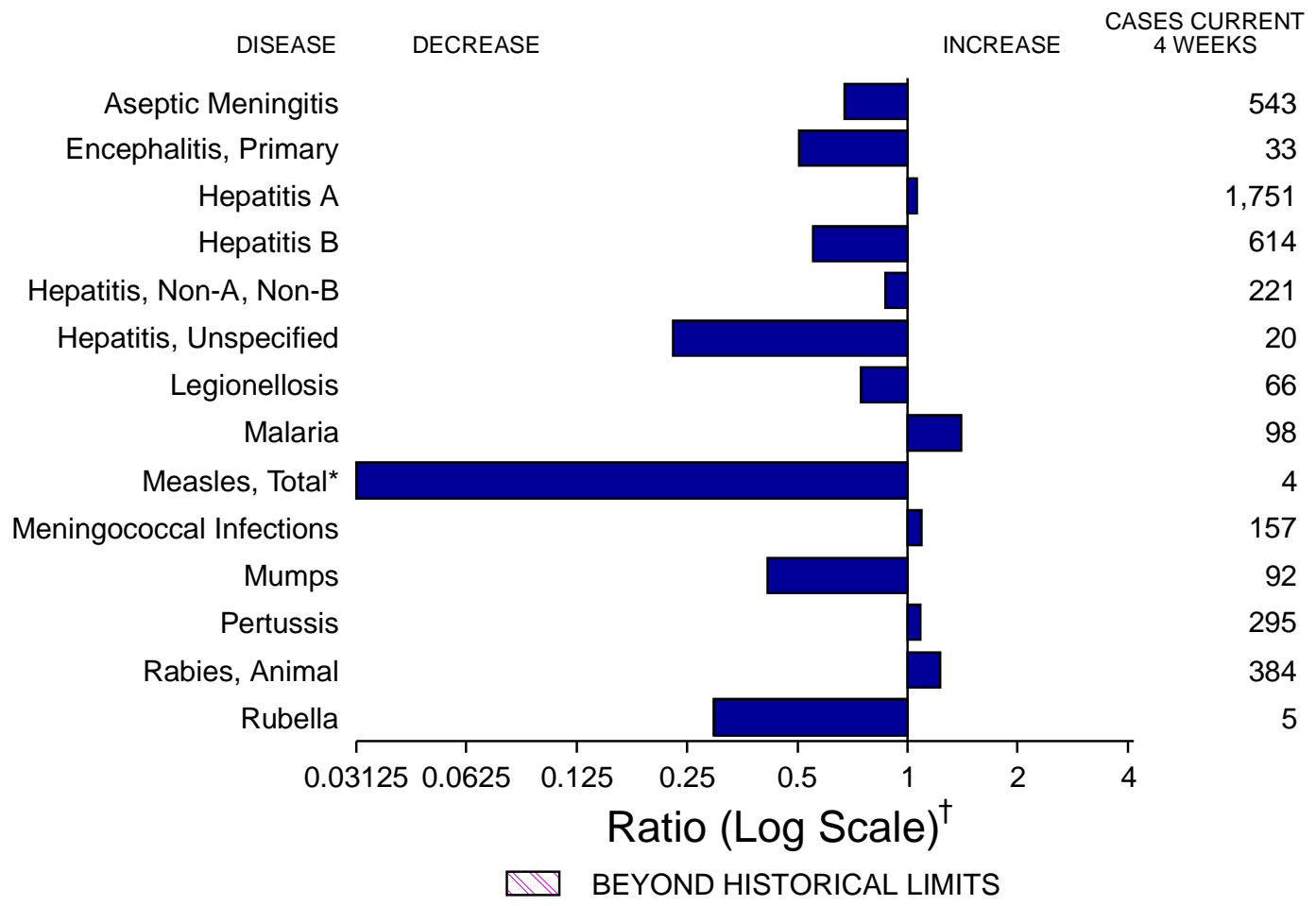

* The large apparent decrease in the number of reported cases of measles (total) reflects dramatic fluctuations in the historical baseline. (Ratio (log scale) for week 50 measles (total) is 0.03125 ).

${ }^{\dagger}$ Ratio of current 4-week total to mean of 15 4-week totals (from previous, comparable, and subsequent 4-week periods for the past 5 years). The point where the hatched area begins is based on the mean and two standard deviations of these 4-week totals.

\section{TABLE I. Summary - cases of specified notifiable diseases, United States, cumulative, week ending December 17, 1994 (50th Week)}

\begin{tabular}{|c|c|c|c|}
\hline & Cum. 1994 & & Cum. 1994 \\
\hline 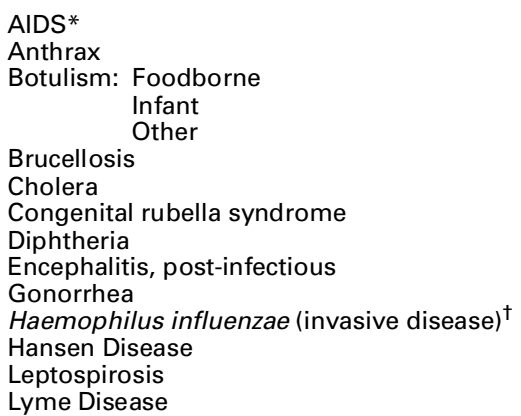 & $\begin{array}{r}72,888 \\
- \\
58 \\
74 \\
7 \\
92 \\
31 \\
6 \\
1 \\
102 \\
378,599 \\
1,081 \\
110 \\
34 \\
11,030\end{array}$ & $\begin{array}{l}\text { Measles: imported } \\
\text { Plague } \\
\text { Poliomyelitis, Paralytic } \S \\
\text { Psittacosis } \\
\text { Rabies, human } \\
\text { Syphilis, primary \& secondary } \\
\text { Syphilis, congenital, age }<1 \text { year } \\
\text { Tetanus } \\
\text { Toxic shock syndrome } \\
\text { Trichinosis } \\
\text { Tuberculosis } \\
\text { Tularemia } \\
\text { Typhoid fever } \\
\text { Typhus fever, tickborne (RMSF) }\end{array}$ & $\begin{array}{r}183 \\
693 \\
14 \\
1 \\
39 \\
2 \\
19,416 \\
1,123 \\
36 \\
176 \\
35 \\
21,126 \\
84 \\
395 \\
434\end{array}$ \\
\hline
\end{tabular}

*Updated monthly to the Division of HIV/AIDS, National Center for Infectious Diseases; last update November $29,1994$.

†Of 1027 cases of known age, $293(29 \%)$ were reported among children less than 5 years of age.

\$This case was vaccine-associated. The remaining 6 suspected cases with onset in 1994 have not yet been confirmed.

ITotal reported to the Division of Sexually Transmitted Diseases and HIV Prevention, National Center for Prevention Services through second quarter 1994. 
TABLE II. Cases of selected notifiable diseases, United States, weeks ending December 17, 1994, and December 18, 1993 (50th Week)

\begin{tabular}{|c|c|c|c|c|c|c|c|c|c|c|c|c|}
\hline \multirow{3}{*}{ Reporting Area } & \multirow{3}{*}{\begin{tabular}{|c|} 
AIDS* \\
Cum. \\
1994 \\
\end{tabular}} & \multirow{3}{*}{$\begin{array}{c}\text { Aseptic } \\
\text { Menin- } \\
\text { gitis } \\
\text { Cum. } \\
1994 \\
\end{array}$} & \multicolumn{2}{|c|}{ Encephalitis } & \multirow{2}{*}{\multicolumn{2}{|c|}{ Gonorrhea }} & \multicolumn{4}{|c|}{ Hepatitis (Viral), by type } & \multirow{3}{*}{$\begin{array}{c}\begin{array}{c}\text { Legionel- } \\
\text { losis }\end{array} \\
\text { Cum. } \\
1994 \\
\end{array}$} & \multirow{3}{*}{$\begin{array}{c}\begin{array}{c}\text { Lyme } \\
\text { Disease }\end{array} \\
\text { Cum. } \\
1994 \\
\end{array}$} \\
\hline & & & Primary & $\begin{array}{l}\text { Post-in- } \\
\text { fectious }\end{array}$ & & & \multirow{2}{*}{$\begin{array}{c}\text { A } \\
\text { Cum. } \\
1994\end{array}$} & \multirow{2}{*}{\begin{tabular}{|c|} 
B \\
Cum. \\
1994 \\
\end{tabular}} & \multirow{2}{*}{$\begin{array}{c}\text { NA,NB } \\
\text { Cum. } \\
1994\end{array}$} & \multirow{2}{*}{$\begin{array}{c}\begin{array}{c}\text { Unspeci- } \\
\text { fied }\end{array} \\
\text { Cum. } \\
1994 \\
\end{array}$} & & \\
\hline & & & $\begin{array}{l}\text { Cum. } \\
1994\end{array}$ & $\begin{array}{l}\text { Cum. } \\
1994\end{array}$ & $\begin{array}{l}\text { Cum. } \\
1994\end{array}$ & $\begin{array}{l}\text { Cum. } \\
1993\end{array}$ & & & & & & \\
\hline UNITED STATES & 72,888 & 7,804 & 634 & 102 & 378,599 & 385,032 & 22,407 & 10,986 & 4,106 & 398 & 1,499 & 11,030 \\
\hline $\begin{array}{l}\text { NEW ENGLAND } \\
\text { Maine } \\
\text { N.H. } \\
\text { Vt. } \\
\text { Mass. } \\
\text { R.I. } \\
\text { Conn. }\end{array}$ & $\begin{array}{r}2,589 \\
79 \\
60 \\
34 \\
1,309 \\
241 \\
866\end{array}$ & $\begin{array}{r}308 \\
31 \\
33 \\
36 \\
88 \\
120 \\
-\end{array}$ & $\begin{array}{r}18 \\
5 \\
- \\
3 \\
8 \\
2 \\
-\end{array}$ & $\begin{array}{l}5 \\
- \\
2 \\
- \\
1 \\
2 \\
-\end{array}$ & $\begin{array}{r}7,779 \\
87 \\
105 \\
35 \\
3,130 \\
457 \\
3,965\end{array}$ & $\begin{array}{r}7,561 \\
77 \\
71 \\
24 \\
3,076 \\
417 \\
3,896\end{array}$ & $\begin{array}{r}279 \\
24 \\
15 \\
14 \\
102 \\
27 \\
97\end{array}$ & $\begin{array}{r}305 \\
11 \\
22 \\
- \\
175 \\
8 \\
89\end{array}$ & $\begin{array}{r}124 \\
- \\
8 \\
- \\
96 \\
20 \\
-\end{array}$ & $\begin{array}{r}15 \\
- \\
- \\
- \\
13 \\
2 \\
-\end{array}$ & $\begin{array}{r}76 \\
5 \\
- \\
1 \\
58 \\
12 \\
-\end{array}$ & $\begin{array}{r}2,547 \\
27 \\
31 \\
13 \\
249 \\
471 \\
1,756\end{array}$ \\
\hline $\begin{array}{l}\text { MID. ATLANTIC } \\
\text { Upstate N.Y. } \\
\text { N.Y. City } \\
\text { N.J. } \\
\text { Pa. }\end{array}$ & $\begin{array}{r}21,304 \\
2,006 \\
12,177 \\
4,655 \\
2,466\end{array}$ & $\begin{array}{r}905 \\
443 \\
141 \\
- \\
321\end{array}$ & $\begin{array}{r}60 \\
35 \\
7 \\
- \\
18\end{array}$ & $\begin{array}{r}19 \\
3 \\
5 \\
- \\
11\end{array}$ & $\begin{array}{r}41,674 \\
9,995 \\
14,236 \\
5,089 \\
12,354\end{array}$ & $\begin{array}{r}45,367 \\
9,963 \\
12,294 \\
5,558 \\
17,552\end{array}$ & $\begin{array}{r}1,607 \\
519 \\
636 \\
278 \\
174\end{array}$ & $\begin{array}{r}1,426 \\
375 \\
360 \\
356 \\
335\end{array}$ & $\begin{array}{r}443 \\
217 \\
4 \\
187 \\
35\end{array}$ & $\begin{array}{l}9 \\
5 \\
- \\
- \\
4\end{array}$ & $\begin{array}{r}244 \\
59 \\
10 \\
41 \\
134\end{array}$ & $\begin{array}{r}6,953 \\
4,312 \\
28 \\
1,303 \\
1,310\end{array}$ \\
\hline $\begin{array}{l}\text { E.N. CENTRAL } \\
\text { Ohio } \\
\text { Ind. } \\
\text { Ill. } \\
\text { Mich. } \\
\text { Wis. }\end{array}$ & $\begin{array}{r}5,883 \\
1,095 \\
589 \\
2,896 \\
960 \\
343\end{array}$ & $\begin{array}{r}1,495 \\
384 \\
197 \\
382 \\
525 \\
7\end{array}$ & $\begin{array}{r}160 \\
55 \\
12 \\
53 \\
36 \\
4\end{array}$ & $\begin{array}{r}22 \\
4 \\
1 \\
5 \\
12 \\
-\end{array}$ & $\begin{array}{r}74,452 \\
22,239 \\
8,766 \\
19,040 \\
17,501 \\
6,906\end{array}$ & $\begin{array}{r}82,179 \\
22,021 \\
8,328 \\
27,871 \\
17,606 \\
6,353\end{array}$ & $\begin{array}{r}2,408 \\
1,106 \\
359 \\
447 \\
334 \\
162\end{array}$ & $\begin{array}{r}1,089 \\
161 \\
175 \\
222 \\
406 \\
125\end{array}$ & $\begin{array}{r}300 \\
23 \\
10 \\
64 \\
200 \\
3\end{array}$ & $\begin{array}{r}12 \\
- \\
- \\
5 \\
7 \\
-\end{array}$ & $\begin{array}{r}436 \\
192 \\
105 \\
30 \\
79 \\
30\end{array}$ & $\begin{array}{r}134 \\
76 \\
14 \\
11 \\
33 \\
-\end{array}$ \\
\hline $\begin{array}{l}\text { W.N. CENTRAL } \\
\text { Minn. } \\
\text { lowa } \\
\text { Mo. } \\
\text { N. Dak. } \\
\text { S. Dak. } \\
\text { Nebr. } \\
\text { Kans. }\end{array}$ & $\begin{array}{r}1,502 \\
375 \\
96 \\
671 \\
22 \\
15 \\
84 \\
239\end{array}$ & $\begin{array}{r}422 \\
25 \\
118 \\
152 \\
13 \\
2 \\
37 \\
75\end{array}$ & $\begin{array}{r}34 \\
5 \\
1 \\
8 \\
4 \\
4 \\
5 \\
7\end{array}$ & $\begin{array}{l}8 \\
- \\
1 \\
4 \\
- \\
- \\
3 \\
-\end{array}$ & $\begin{array}{r}21,520 \\
3,403 \\
1,576 \\
11,609 \\
34 \\
197 \\
1,060 \\
3,641\end{array}$ & $\begin{array}{r}20,890 \\
2,381 \\
1,508 \\
12,551 \\
52 \\
245 \\
484 \\
3,669\end{array}$ & $\begin{array}{r}1,118 \\
231 \\
64 \\
563 \\
5 \\
37 \\
118 \\
100\end{array}$ & $\begin{array}{r}639 \\
64 \\
26 \\
486 \\
1 \\
4 \\
4 \\
29 \\
29\end{array}$ & $\begin{array}{r}107 \\
23 \\
13 \\
42 \\
- \\
- \\
14 \\
15\end{array}$ & $\begin{array}{r}12 \\
1 \\
11 \\
- \\
- \\
- \\
- \\
-\end{array}$ & $\begin{array}{r}94 \\
2 \\
33 \\
38 \\
4 \\
1 \\
10 \\
10 \\
6\end{array}$ & $\begin{array}{r}285 \\
165 \\
17 \\
87 \\
- \\
- \\
2 \\
14\end{array}$ \\
\hline $\begin{array}{l}\text { S. ATLANTIC } \\
\text { Del. } \\
\text { Md. } \\
\text { D.C. } \\
\text { Va. } \\
\text { W. Va. } \\
\text { N.C. } \\
\text { S.C. } \\
\text { Ga. } \\
\text { Fla. }\end{array}$ & $\begin{array}{r}17,469 \\
247 \\
2,526 \\
1,325 \\
1,089 \\
76 \\
1,152 \\
1,088 \\
2,071 \\
7,895\end{array}$ & $\begin{array}{r}1,533 \\
37 \\
248 \\
53 \\
313 \\
39 \\
218 \\
31 \\
50 \\
544\end{array}$ & $\begin{array}{r}143 \\
1 \\
22 \\
- \\
31 \\
48 \\
40 \\
- \\
1 \\
-\end{array}$ & $\begin{array}{r}30 \\
- \\
4 \\
1 \\
6 \\
- \\
1 \\
- \\
- \\
18\end{array}$ & $\begin{array}{r}106,379 \\
1,892 \\
17,202 \\
6,852 \\
13,180 \\
806 \\
27,052 \\
12,518 \\
3,899 \\
22,978\end{array}$ & $\begin{array}{r}96,631 \\
1,507 \\
16,000 \\
5,384 \\
11,889 \\
670 \\
23,941 \\
10,197 \\
4,660 \\
22,383\end{array}$ & $\begin{array}{r}1,414 \\
17 \\
214 \\
29 \\
184 \\
21 \\
139 \\
39 \\
33 \\
738\end{array}$ & $\begin{array}{r}2,212 \\
5 \\
399 \\
56 \\
129 \\
45 \\
276 \\
33 \\
532 \\
737\end{array}$ & $\begin{array}{r}606 \\
1 \\
32 \\
2 \\
25 \\
44 \\
53 \\
10 \\
185 \\
254\end{array}$ & $\begin{array}{r}55 \\
- \\
16 \\
- \\
10 \\
- \\
- \\
- \\
- \\
29\end{array}$ & $\begin{array}{r}336 \\
26 \\
89 \\
10 \\
12 \\
4 \\
27 \\
16 \\
99 \\
53\end{array}$ & $\begin{array}{r}839 \\
78 \\
379 \\
9 \\
129 \\
27 \\
77 \\
7 \\
106 \\
27\end{array}$ \\
\hline $\begin{array}{l}\text { E.S. CENTRAL } \\
\text { Ky. } \\
\text { Tenn. } \\
\text { Ala. } \\
\text { Miss. }\end{array}$ & $\begin{array}{r}1,942 \\
296 \\
693 \\
554 \\
399\end{array}$ & $\begin{array}{r}514 \\
178 \\
124 \\
163 \\
49\end{array}$ & $\begin{array}{r}39 \\
16 \\
12 \\
8 \\
3\end{array}$ & $\begin{array}{l}3 \\
1 \\
- \\
1 \\
1\end{array}$ & $\begin{array}{r}44,384 \\
5,008 \\
14,346 \\
14,133 \\
10,897\end{array}$ & $\begin{array}{r}44,169 \\
4,810 \\
13,660 \\
15,735 \\
9,964\end{array}$ & $\begin{array}{r}659 \\
186 \\
279 \\
122 \\
72\end{array}$ & $\begin{array}{r}1,110 \\
70 \\
953 \\
87 \\
-\end{array}$ & $\begin{array}{r}890 \\
32 \\
838 \\
20 \\
-\end{array}$ & $\begin{array}{l}2 \\
- \\
1 \\
1 \\
-\end{array}$ & $\begin{array}{r}77 \\
9 \\
44 \\
13 \\
11\end{array}$ & $\begin{array}{r}42 \\
23 \\
13 \\
6 \\
-\end{array}$ \\
\hline $\begin{array}{l}\text { W.S. CENTRAL } \\
\text { Ark. } \\
\text { La. } \\
\text { Okla. } \\
\text { Tex. }\end{array}$ & $\begin{array}{r}6,982 \\
255 \\
1,146 \\
244 \\
5,337\end{array}$ & $\begin{array}{r}854 \\
50 \\
34 \\
- \\
770\end{array}$ & $\begin{array}{r}50 \\
- \\
8 \\
- \\
42\end{array}$ & $\begin{array}{l}2 \\
- \\
- \\
- \\
2\end{array}$ & $\begin{array}{r}45,650 \\
6,406 \\
11,555 \\
3,259 \\
24,430\end{array}$ & $\begin{array}{r}43,187 \\
7,403 \\
11,560 \\
4,437 \\
19,787\end{array}$ & $\begin{array}{r}3,247 \\
202 \\
151 \\
366 \\
2,528\end{array}$ & $\begin{array}{r}1,509 \\
37 \\
167 \\
303 \\
1,002\end{array}$ & $\begin{array}{r}595 \\
8 \\
181 \\
337 \\
69\end{array}$ & $\begin{array}{r}71 \\
3 \\
1 \\
3 \\
64\end{array}$ & $\begin{array}{l}46 \\
10 \\
14 \\
11 \\
11\end{array}$ & $\begin{array}{r}128 \\
8 \\
2 \\
73 \\
45\end{array}$ \\
\hline $\begin{array}{l}\text { MOUNTAIN } \\
\text { Mont. } \\
\text { Idaho } \\
\text { Wyo. } \\
\text { Colo. } \\
\text { N. Mex. } \\
\text { Ariz. } \\
\text { Utah } \\
\text { Nev. }\end{array}$ & $\begin{array}{r}2,107 \\
26 \\
56 \\
18 \\
763 \\
198 \\
559 \\
131 \\
356\end{array}$ & $\begin{array}{r}343 \\
8 \\
6 \\
4 \\
134 \\
19 \\
74 \\
55 \\
43\end{array}$ & $\begin{array}{r}12 \\
- \\
- \\
3 \\
3 \\
- \\
- \\
2 \\
4\end{array}$ & $\begin{array}{l}4 \\
- \\
- \\
1 \\
- \\
- \\
2 \\
1 \\
-\end{array}$ & $\begin{array}{r}9,480 \\
84 \\
92 \\
85 \\
3,237 \\
1,066 \\
3,084 \\
232 \\
1,600\end{array}$ & $\begin{array}{r}10,841 \\
84 \\
169 \\
75 \\
3,666 \\
933 \\
3,675 \\
413 \\
1,826\end{array}$ & $\begin{array}{r}4,330 \\
24 \\
366 \\
30 \\
575 \\
1,101 \\
1,401 \\
599 \\
234\end{array}$ & $\begin{array}{r}622 \\
23 \\
74 \\
23 \\
99 \\
212 \\
56 \\
78 \\
57\end{array}$ & $\begin{array}{r}424 \\
15 \\
68 \\
166 \\
73 \\
46 \\
17 \\
20 \\
19\end{array}$ & $\begin{array}{r}62 \\
- \\
1 \\
- \\
17 \\
11 \\
15 \\
4 \\
14\end{array}$ & $\begin{array}{r}105 \\
16 \\
2 \\
6 \\
21 \\
4 \\
25 \\
7 \\
24\end{array}$ & $\begin{array}{r}20 \\
- \\
3 \\
5 \\
- \\
8 \\
- \\
3 \\
1\end{array}$ \\
\hline $\begin{array}{l}\text { PACIFIC } \\
\text { Wash. } \\
\text { Oreg. } \\
\text { Calif. } \\
\text { Alaska } \\
\text { Hawaii }\end{array}$ & $\begin{array}{r}13,110 \\
856 \\
550 \\
11,481 \\
40 \\
183\end{array}$ & $\begin{array}{r}1,430 \\
- \\
- \\
1,281 \\
18 \\
131\end{array}$ & $\begin{array}{r}118 \\
- \\
- \\
115 \\
3 \\
-\end{array}$ & $\begin{array}{l}9 \\
- \\
- \\
8 \\
- \\
1\end{array}$ & $\begin{array}{r}27,281 \\
2,783 \\
571 \\
22,465 \\
864 \\
598\end{array}$ & $\begin{array}{r}34,207 \\
3,600 \\
1,122 \\
28,255 \\
626 \\
604\end{array}$ & $\begin{array}{r}7,345 \\
337 \\
803 \\
5,946 \\
202 \\
57\end{array}$ & $\begin{array}{r}2,074 \\
74 \\
85 \\
1,876 \\
11 \\
28\end{array}$ & $\begin{array}{r}617 \\
72 \\
22 \\
518 \\
- \\
5\end{array}$ & $\begin{array}{r}160 \\
2 \\
1 \\
154 \\
- \\
3\end{array}$ & $\begin{array}{r}85 \\
8 \\
- \\
73 \\
- \\
4\end{array}$ & $\begin{array}{r}82 \\
- \\
- \\
82 \\
- \\
-\end{array}$ \\
\hline $\begin{array}{l}\text { Guam } \\
\text { P.R. } \\
\text { V.I. } \\
\text { Amer. Samoa } \\
\text { C.N.M.I. }\end{array}$ & $\begin{array}{r}1 \\
2,159 \\
49 \\
- \\
-\end{array}$ & $\begin{array}{r}22 \\
39 \\
- \\
- \\
-\end{array}$ & $\begin{array}{l}- \\
1 \\
- \\
- \\
-\end{array}$ & $\begin{array}{l}- \\
3 \\
- \\
- \\
-\end{array}$ & $\begin{array}{r}197 \\
445 \\
41 \\
31 \\
45\end{array}$ & $\begin{array}{r}98 \\
474 \\
91 \\
41 \\
78\end{array}$ & $\begin{array}{r}44 \\
84 \\
- \\
8 \\
8\end{array}$ & $\begin{array}{r}6 \\
358 \\
1 \\
- \\
1\end{array}$ & $\begin{array}{r}1 \\
169 \\
- \\
- \\
-\end{array}$ & $\begin{array}{r}12 \\
11 \\
- \\
- \\
-\end{array}$ & $\begin{array}{l}3 \\
- \\
- \\
- \\
-\end{array}$ & $\begin{array}{l}- \\
- \\
- \\
-\end{array}$ \\
\hline
\end{tabular}

$\mathrm{N}$ : Not notifiable U: Unavailable

C.N.M.I.: Commonwealth of Northern Mariana Islands

*Updated monthly to the Division of HIV/AIDS, National Center for Infectious Diseases; last update November $29,1994$. 
TABLE II. (Cont'd.) Cases of selected notifiable diseases, United States, weeks ending December 17, 1994, and December 18, 1993 (50th Week)

\begin{tabular}{|c|c|c|c|c|c|c|c|c|c|c|c|c|c|c|c|}
\hline \multirow{3}{*}{ Reporting Area } & \multirow{3}{*}{\begin{tabular}{|c|} 
Malaria \\
Cum. \\
1994 \\
\end{tabular}} & \multicolumn{5}{|c|}{ Measles (Rubeola) } & \multirow{3}{*}{\begin{tabular}{|c|}
$\begin{array}{c}\text { Menin- } \\
\text { gococcal } \\
\text { Infections }\end{array}$ \\
Cum. \\
1994 \\
\end{tabular}} & \multirow{2}{*}{\multicolumn{2}{|c|}{ Mumps }} & \multirow{2}{*}{\multicolumn{3}{|c|}{ Pertussis }} & \multirow{2}{*}{\multicolumn{3}{|c|}{ Rubella }} \\
\hline & & \multicolumn{2}{|c|}{ Indigenous } & \multicolumn{2}{|c|}{ Imported* } & \multirow{2}{*}{\begin{tabular}{|c|} 
Total \\
Cum. \\
1993 \\
\end{tabular}} & & & & & & & & & \\
\hline & & 1994 & $\begin{array}{l}\text { Cum. } \\
1994 \\
\end{array}$ & 1994 & $\begin{array}{c}\text { Cum. } \\
1994\end{array}$ & & & 1994 & $\begin{array}{l}\text { Cum. } \\
1994\end{array}$ & 1994 & $\begin{array}{c}\text { Cum. } \\
1994\end{array}$ & $\begin{array}{l}\text { Cum. } \\
1993\end{array}$ & 1994 & $\begin{array}{l}\text { Cum. } \\
1994 \\
\end{array}$ & $\begin{array}{l}\text { Cum. } \\
1993\end{array}$ \\
\hline UNITED STATES & 1,036 & 3 & 693 & - & 183 & 304 & 2,527 & 20 & 1,355 & 120 & 3,484 & 6,087 & 2 & 219 & 177 \\
\hline $\begin{array}{l}\text { NEW ENGLAND } \\
\text { Maine } \\
\text { N.H. } \\
\text { Vt. } \\
\text { Mass. } \\
\text { R.I. } \\
\text { Conn. }\end{array}$ & $\begin{array}{r}77 \\
6 \\
3 \\
3 \\
34 \\
9 \\
22\end{array}$ & $\begin{array}{l}- \\
- \\
- \\
- \\
- \\
-\end{array}$ & $\begin{array}{r}14 \\
1 \\
1 \\
2 \\
2 \\
4 \\
4\end{array}$ & $\begin{array}{l}- \\
- \\
- \\
- \\
- \\
- \\
-\end{array}$ & $\begin{array}{r}14 \\
4 \\
- \\
1 \\
6 \\
3 \\
-\end{array}$ & $\begin{array}{r}63 \\
1 \\
2 \\
31 \\
18 \\
2 \\
9\end{array}$ & $\begin{array}{r}137 \\
23 \\
7 \\
4 \\
61 \\
- \\
42\end{array}$ & $\begin{array}{l}1 \\
- \\
- \\
- \\
- \\
1 \\
-\end{array}$ & $\begin{array}{r}26 \\
3 \\
4 \\
- \\
3 \\
4 \\
12\end{array}$ & $\begin{array}{r}20 \\
- \\
2 \\
- \\
17 \\
- \\
1\end{array}$ & $\begin{array}{r}449 \\
18 \\
84 \\
45 \\
255 \\
7 \\
40\end{array}$ & $\begin{array}{r}739 \\
18 \\
155 \\
91 \\
377 \\
14 \\
84\end{array}$ & $\begin{array}{l}- \\
- \\
- \\
- \\
- \\
-\end{array}$ & $\begin{array}{r}131 \\
- \\
- \\
- \\
125 \\
3 \\
3\end{array}$ & $\begin{array}{l}2 \\
1 \\
- \\
- \\
1 \\
- \\
-\end{array}$ \\
\hline $\begin{array}{l}\text { MID. ATLANTIC } \\
\text { Upstate N.Y. } \\
\text { N.Y. City } \\
\text { N.J. } \\
\text { Pa. }\end{array}$ & $\begin{array}{r}213 \\
56 \\
79 \\
48 \\
30\end{array}$ & $\begin{array}{l}- \\
- \\
- \\
-\end{array}$ & $\begin{array}{r}172 \\
12 \\
11 \\
144 \\
5\end{array}$ & $\begin{array}{l}- \\
- \\
- \\
-\end{array}$ & $\begin{array}{r}33 \\
14 \\
3 \\
12 \\
4\end{array}$ & $\begin{array}{r}38 \\
8 \\
19 \\
11 \\
-\end{array}$ & $\begin{array}{r}249 \\
91 \\
11 \\
55 \\
92\end{array}$ & $\begin{array}{l}- \\
- \\
- \\
-\end{array}$ & $\begin{array}{r}108 \\
32 \\
13 \\
7 \\
56\end{array}$ & $\begin{array}{l}3 \\
3 \\
- \\
- \\
-\end{array}$ & $\begin{array}{r}592 \\
230 \\
157 \\
11 \\
194\end{array}$ & $\begin{array}{r}931 \\
338 \\
91 \\
85 \\
417\end{array}$ & $\begin{array}{l}- \\
- \\
- \\
-\end{array}$ & $\begin{array}{r}10 \\
8 \\
1 \\
1 \\
-\end{array}$ & $\begin{array}{r}59 \\
17 \\
22 \\
15 \\
5\end{array}$ \\
\hline $\begin{array}{l}\text { E.N. CENTRAL } \\
\text { Ohio } \\
\text { Ind. } \\
\text { Ill. } \\
\text { Mich. } \\
\text { Wis. }\end{array}$ & $\begin{array}{r}98 \\
15 \\
14 \\
39 \\
28 \\
2\end{array}$ & $\begin{array}{c}1 \\
- \\
U \\
- \\
1 \\
-\end{array}$ & $\begin{array}{r}59 \\
15 \\
- \\
17 \\
24 \\
3\end{array}$ & $\begin{array}{l}- \\
- \\
\\
- \\
-\end{array}$ & $\begin{array}{r}44 \\
2 \\
1 \\
39 \\
2 \\
-\end{array}$ & $\begin{array}{r}31 \\
9 \\
1 \\
9 \\
6 \\
6\end{array}$ & $\begin{array}{r}403 \\
117 \\
76 \\
117 \\
58 \\
35\end{array}$ & $\begin{array}{r}12 \\
3 \\
U \\
- \\
9 \\
-\end{array}$ & $\begin{array}{r}257 \\
74 \\
7 \\
106 \\
56 \\
14\end{array}$ & $\begin{array}{l}7 \\
7 \\
U \\
- \\
- \\
-\end{array}$ & $\begin{array}{r}414 \\
157 \\
65 \\
94 \\
48 \\
50\end{array}$ & $\begin{array}{r}1,503 \\
434 \\
165 \\
426 \\
115 \\
363\end{array}$ & $\begin{array}{c}1 \\
- \\
U \\
- \\
1 \\
-\end{array}$ & $\begin{array}{r}12 \\
- \\
- \\
3 \\
9 \\
-\end{array}$ & $\begin{array}{l}8 \\
1 \\
3 \\
1 \\
2 \\
1\end{array}$ \\
\hline $\begin{array}{l}\text { W.N. CENTRAL } \\
\text { Minn. } \\
\text { lowa } \\
\text { Mo. } \\
\text { N. Dak. } \\
\text { S. Dak. } \\
\text { Nebr. } \\
\text { Kans. }\end{array}$ & $\begin{array}{r}45 \\
14 \\
5 \\
13 \\
1 \\
- \\
5 \\
7\end{array}$ & $\begin{array}{l}- \\
- \\
- \\
- \\
- \\
- \\
-\end{array}$ & $\begin{array}{r}126 \\
- \\
6 \\
118 \\
- \\
- \\
1 \\
1\end{array}$ & $\begin{array}{l}- \\
- \\
- \\
- \\
- \\
- \\
- \\
-\end{array}$ & $\begin{array}{r}44 \\
- \\
1 \\
42 \\
- \\
- \\
1 \\
-\end{array}$ & $\begin{array}{l}3 \\
- \\
- \\
1 \\
- \\
- \\
- \\
2\end{array}$ & $\begin{array}{r}176 \\
18 \\
20 \\
87 \\
1 \\
9 \\
13 \\
28\end{array}$ & $\begin{array}{l}- \\
- \\
- \\
- \\
- \\
- \\
-\end{array}$ & $\begin{array}{r}66 \\
5 \\
16 \\
39 \\
5 \\
- \\
1 \\
-\end{array}$ & $\begin{array}{r}17 \\
13 \\
2 \\
- \\
- \\
2 \\
- \\
-\end{array}$ & $\begin{array}{r}222 \\
100 \\
23 \\
44 \\
5 \\
26 \\
11 \\
13\end{array}$ & $\begin{array}{r}545 \\
319 \\
37 \\
140 \\
5 \\
8 \\
14 \\
22\end{array}$ & $\begin{array}{l}- \\
- \\
- \\
- \\
- \\
- \\
-\end{array}$ & $\begin{array}{l}2 \\
- \\
- \\
2 \\
- \\
- \\
- \\
-\end{array}$ & $\begin{array}{l}1 \\
- \\
- \\
1 \\
- \\
- \\
- \\
-\end{array}$ \\
\hline $\begin{array}{l}\text { S. ATLANTIC } \\
\text { Del. } \\
\text { Md. } \\
\text { D.C. } \\
\text { Va. } \\
\text { W. Va. } \\
\text { N.C. } \\
\text { S.C. } \\
\text { Ga. } \\
\text { Fla. }\end{array}$ & $\begin{array}{r}224 \\
3 \\
99 \\
15 \\
37 \\
- \\
11 \\
5 \\
26 \\
28\end{array}$ & $\begin{array}{l}- \\
\text { U } \\
- \\
- \\
- \\
- \\
- \\
- \\
-\end{array}$ & $\begin{array}{r}59 \\
- \\
2 \\
- \\
1 \\
36 \\
2 \\
- \\
3 \\
15\end{array}$ & $\begin{array}{l}- \\
\text { U } \\
- \\
- \\
- \\
- \\
- \\
- \\
-\end{array}$ & $\begin{array}{l}8 \\
- \\
2 \\
- \\
2 \\
- \\
1 \\
- \\
- \\
3\end{array}$ & $\begin{array}{r}29 \\
- \\
4 \\
- \\
4 \\
- \\
1 \\
- \\
- \\
20\end{array}$ & $\begin{array}{r}431 \\
5 \\
44 \\
6 \\
66 \\
14 \\
53 \\
33 \\
69 \\
141\end{array}$ & $\begin{array}{l}2 \\
U \\
- \\
- \\
2 \\
- \\
- \\
- \\
- \\
-\end{array}$ & $\begin{array}{r}201 \\
- \\
68 \\
- \\
44 \\
3 \\
36 \\
8 \\
9 \\
33\end{array}$ & $\begin{array}{l}1 \\
U \\
- \\
- \\
- \\
- \\
- \\
- \\
- \\
1\end{array}$ & $\begin{array}{r}359 \\
3 \\
77 \\
10 \\
36 \\
5 \\
140 \\
14 \\
27 \\
47\end{array}$ & $\begin{array}{r}646 \\
10 \\
131 \\
14 \\
65 \\
8 \\
195 \\
73 \\
54 \\
96\end{array}$ & $\begin{array}{l}- \\
\\
- \\
- \\
- \\
- \\
- \\
- \\
-\end{array}$ & $\begin{array}{r}11 \\
- \\
- \\
- \\
- \\
- \\
- \\
- \\
2 \\
9\end{array}$ & $\begin{array}{l}7 \\
- \\
3 \\
- \\
- \\
- \\
- \\
- \\
- \\
4\end{array}$ \\
\hline $\begin{array}{l}\text { E.S. CENTRAL } \\
\text { Ky. } \\
\text { Tenn. } \\
\text { Ala. } \\
\text { Miss. }\end{array}$ & $\begin{array}{r}32 \\
12 \\
10 \\
9 \\
1\end{array}$ & $\begin{array}{l}- \\
- \\
- \\
-\end{array}$ & $\begin{array}{r}28 \\
- \\
28 \\
- \\
-\end{array}$ & $\begin{array}{l}- \\
- \\
- \\
-\end{array}$ & $\begin{array}{l}- \\
- \\
- \\
-\end{array}$ & $\begin{array}{l}1 \\
- \\
- \\
1 \\
-\end{array}$ & $\begin{array}{r}148 \\
38 \\
35 \\
75 \\
-\end{array}$ & $\begin{array}{l}- \\
- \\
- \\
-\end{array}$ & $\begin{array}{r}27 \\
- \\
8 \\
12 \\
7\end{array}$ & $\begin{array}{l}- \\
- \\
- \\
-\end{array}$ & $\begin{array}{r}122 \\
59 \\
22 \\
34 \\
7\end{array}$ & $\begin{array}{r}281 \\
38 \\
172 \\
60 \\
11\end{array}$ & $\begin{array}{l}- \\
- \\
- \\
-\end{array}$ & $\begin{array}{l}- \\
- \\
- \\
-\end{array}$ & $\begin{array}{l}1 \\
1 \\
- \\
- \\
-\end{array}$ \\
\hline $\begin{array}{l}\text { W.S. CENTRAL } \\
\text { Ark. } \\
\text { La. } \\
\text { Okla. } \\
\text { Tex. }\end{array}$ & $\begin{array}{r}75 \\
3 \\
10 \\
7 \\
55\end{array}$ & $\begin{array}{l}- \\
- \\
- \\
-\end{array}$ & $\begin{array}{r}11 \\
- \\
- \\
- \\
11\end{array}$ & $\begin{array}{l}- \\
- \\
- \\
- \\
-\end{array}$ & $\begin{array}{l}8 \\
1 \\
1 \\
- \\
6\end{array}$ & $\begin{array}{r}10 \\
- \\
1 \\
- \\
9\end{array}$ & $\begin{array}{r}325 \\
44 \\
38 \\
33 \\
210\end{array}$ & $\begin{array}{l}2 \\
- \\
2 \\
- \\
-\end{array}$ & $\begin{array}{r}249 \\
5 \\
34 \\
23 \\
187\end{array}$ & $\begin{array}{l}5 \\
- \\
- \\
5 \\
-\end{array}$ & $\begin{array}{r}192 \\
27 \\
12 \\
32 \\
121\end{array}$ & $\begin{array}{r}187 \\
12 \\
14 \\
78 \\
83\end{array}$ & $\begin{array}{l}- \\
- \\
- \\
-\end{array}$ & $\begin{array}{r}13 \\
- \\
- \\
4 \\
9\end{array}$ & $\begin{array}{r}18 \\
- \\
1 \\
1 \\
16\end{array}$ \\
\hline $\begin{array}{l}\text { MOUNTAIN } \\
\text { Mont. } \\
\text { Idaho } \\
\text { Wyo. } \\
\text { Colo. } \\
\text { N. Mex. } \\
\text { Ariz. } \\
\text { Utah } \\
\text { Nev. }\end{array}$ & $\begin{array}{r}39 \\
- \\
2 \\
1 \\
18 \\
3 \\
9 \\
4 \\
2\end{array}$ & $\begin{array}{l}2 \\
- \\
- \\
- \\
1 \\
- \\
1 \\
- \\
-\end{array}$ & $\begin{array}{r}152 \\
- \\
1 \\
- \\
17 \\
- \\
3 \\
131 \\
-\end{array}$ & $\begin{array}{l}- \\
- \\
- \\
- \\
- \\
- \\
- \\
- \\
-\end{array}$ & $\begin{array}{r}17 \\
- \\
- \\
- \\
3 \\
- \\
1 \\
2 \\
11\end{array}$ & $\begin{array}{l}7 \\
- \\
- \\
- \\
3 \\
- \\
3 \\
- \\
1\end{array}$ & $\begin{array}{r}164 \\
6 \\
17 \\
9 \\
38 \\
15 \\
48 \\
19 \\
12\end{array}$ & $\begin{array}{r}1 \\
- \\
- \\
- \\
- \\
N \\
- \\
1 \\
-\end{array}$ & $\begin{array}{r}153 \\
- \\
10 \\
3 \\
3 \\
\mathrm{~N} \\
95 \\
26 \\
15\end{array}$ & $\begin{array}{r}65 \\
1 \\
63 \\
- \\
1 \\
- \\
- \\
- \\
-\end{array}$ & $\begin{array}{r}477 \\
11 \\
150 \\
- \\
125 \\
33 \\
131 \\
24 \\
3\end{array}$ & $\begin{array}{r}444 \\
11 \\
99 \\
1 \\
184 \\
39 \\
69 \\
36 \\
5\end{array}$ & $\begin{array}{l}- \\
- \\
- \\
- \\
- \\
- \\
- \\
-\end{array}$ & $\begin{array}{l}5 \\
- \\
- \\
- \\
- \\
- \\
- \\
4 \\
1\end{array}$ & $\begin{array}{r}11 \\
- \\
2 \\
- \\
2 \\
- \\
2 \\
4 \\
1\end{array}$ \\
\hline $\begin{array}{l}\text { PACIFIC } \\
\text { Wash. } \\
\text { Oreg. } \\
\text { Calif. } \\
\text { Alaska } \\
\text { Hawaii }\end{array}$ & $\begin{array}{r}233 \\
12 \\
14 \\
190 \\
2 \\
15\end{array}$ & $\begin{array}{l}- \\
- \\
- \\
- \\
-\end{array}$ & $\begin{array}{r}72 \\
- \\
- \\
56 \\
16 \\
-\end{array}$ & $\begin{array}{l}- \\
- \\
- \\
- \\
- \\
-\end{array}$ & $\begin{array}{r}15 \\
- \\
2 \\
9 \\
- \\
4\end{array}$ & $\begin{array}{r}122 \\
- \\
4 \\
96 \\
2 \\
20\end{array}$ & $\begin{array}{r}494 \\
32 \\
100 \\
348 \\
5 \\
9\end{array}$ & $\begin{array}{r}2 \\
- \\
\mathrm{N} \\
2 \\
- \\
-\end{array}$ & $\begin{array}{r}268 \\
8 \\
\mathrm{~N} \\
238 \\
4 \\
18\end{array}$ & $\begin{array}{l}2 \\
1 \\
- \\
1 \\
- \\
-\end{array}$ & $\begin{array}{r}657 \\
33 \\
41 \\
561 \\
1 \\
21\end{array}$ & $\begin{array}{r}811 \\
86 \\
104 \\
604 \\
5 \\
12\end{array}$ & $\begin{array}{l}1 \\
- \\
- \\
1 \\
- \\
-\end{array}$ & $\begin{array}{r}35 \\
- \\
3 \\
27 \\
1 \\
4\end{array}$ & $\begin{array}{r}70 \\
- \\
- \\
41 \\
1 \\
28\end{array}$ \\
\hline $\begin{array}{l}\text { Guam } \\
\text { P.R. } \\
\text { V.I. } \\
\text { Amer. Samoa } \\
\text { C.N.M.I. }\end{array}$ & $\begin{array}{l}4 \\
3 \\
- \\
- \\
1\end{array}$ & $\begin{array}{l}U \\
- \\
- \\
\end{array}$ & $\begin{array}{r}211 \\
13 \\
- \\
- \\
26\end{array}$ & $\begin{array}{l}U \\
- \\
- \\
\bar{U}\end{array}$ & $\begin{array}{l}- \\
- \\
- \\
-\end{array}$ & $\begin{array}{r}17 \\
356 \\
- \\
- \\
63\end{array}$ & $\begin{array}{r}1 \\
15 \\
- \\
- \\
-\end{array}$ & $\begin{array}{l}U \\
- \\
- \\
\end{array}$ & $\begin{array}{l}6 \\
2 \\
1 \\
1 \\
2\end{array}$ & $\begin{array}{l}U \\
- \\
- \\
\bar{U}\end{array}$ & $\begin{array}{l}2 \\
2 \\
- \\
2 \\
-\end{array}$ & $\begin{array}{r}11 \\
- \\
2 \\
1\end{array}$ & $\begin{array}{l}U \\
- \\
- \\
\end{array}$ & $\begin{array}{l}1 \\
- \\
- \\
- \\
-\end{array}$ & $\begin{array}{l}- \\
- \\
- \\
-\end{array}$ \\
\hline
\end{tabular}

*For measles only, imported cases include both out-of-state and international importations.

$\mathrm{N}$ : Not notifiable U: Unavailable $\quad+$ International $\quad$ S Out-of-state 
TABLE II. (Cont'd.) Cases of selected notifiable diseases, United States, weeks ending December 17, 1994, and December 18, 1993 (50th Week)

\begin{tabular}{|c|c|c|c|c|c|c|c|c|c|}
\hline \multirow{2}{*}{ Reporting Area } & \multicolumn{2}{|c|}{$\begin{array}{c}\text { Syphilis } \\
\text { (Primary \& Secondary) }\end{array}$} & \multirow{2}{*}{$\begin{array}{c}\begin{array}{c}\text { Toxic- } \\
\text { Shock } \\
\text { Syndrome }\end{array} \\
\begin{array}{c}\text { Cum. } \\
1994\end{array}\end{array}$} & \multicolumn{2}{|c|}{ Tuberculosis } & \multirow{2}{*}{$\begin{array}{c}\begin{array}{c}\text { Tula- } \\
\text { remia }\end{array} \\
\text { Cum. } \\
1994\end{array}$} & \multirow{2}{*}{$\begin{array}{c}\begin{array}{c}\text { Typhoid } \\
\text { Fever }\end{array} \\
\text { Cum. } \\
1994\end{array}$} & \multirow{2}{*}{$\begin{array}{c}\begin{array}{c}\text { Typhus Fever } \\
\text { (Tick-borne) } \\
\text { (RMSF) }\end{array} \\
\begin{array}{c}\text { Cum. } \\
1994\end{array}\end{array}$} & \multirow{2}{*}{$\begin{array}{l}\begin{array}{l}\text { Rabies, } \\
\text { Animal }\end{array} \\
\text { Cum. } \\
1994\end{array}$} \\
\hline & $\begin{array}{l}\text { Cum. } \\
1994\end{array}$ & $\begin{array}{l}\text { Cum. } \\
1993\end{array}$ & & $\begin{array}{l}\text { Cum. } \\
1994\end{array}$ & $\begin{array}{l}\text { Cum. } \\
1993\end{array}$ & & & & \\
\hline UNITED STATES & 19,416 & 25,322 & 176 & 21,126 & 22,023 & 84 & 395 & 434 & 7,083 \\
\hline $\begin{array}{l}\text { NEW ENGLAND } \\
\text { Maine } \\
\text { N.H. } \\
\text { Vt. } \\
\text { Mass. } \\
\text { R.I. } \\
\text { Conn. }\end{array}$ & $\begin{array}{r}211 \\
4 \\
4 \\
- \\
90 \\
15 \\
98\end{array}$ & $\begin{array}{r}348 \\
8 \\
25 \\
1 \\
122 \\
15 \\
177\end{array}$ & $\begin{array}{l}4 \\
1 \\
- \\
1 \\
2 \\
- \\
-\end{array}$ & $\begin{array}{r}499 \\
27 \\
15 \\
8 \\
8 \\
262 \\
48 \\
139\end{array}$ & $\begin{array}{r}520 \\
25 \\
17 \\
7 \\
291 \\
58 \\
122\end{array}$ & $\begin{array}{l}1 \\
- \\
- \\
- \\
1 \\
- \\
-\end{array}$ & $\begin{array}{r}21 \\
- \\
- \\
17 \\
1 \\
3\end{array}$ & $\begin{array}{c}15 \\
- \\
- \\
- \\
7 \\
- \\
8\end{array}$ & $\begin{array}{r}1,815 \\
- \\
207 \\
140 \\
702 \\
44 \\
722\end{array}$ \\
\hline $\begin{array}{l}\text { MID. ATLANTIC } \\
\text { Upstate N.Y. } \\
\text { N.Y. City } \\
\text { N.J. } \\
\text { Pa. }\end{array}$ & $\begin{array}{r}1,327 \\
162 \\
562 \\
234 \\
369\end{array}$ & $\begin{array}{r}2,330 \\
241 \\
1,183 \\
288 \\
618\end{array}$ & $\begin{array}{r}28 \\
15 \\
- \\
- \\
13\end{array}$ & $\begin{array}{r}4,221 \\
493 \\
2,460 \\
780 \\
488\end{array}$ & $\begin{array}{r}4,746 \\
661 \\
2,693 \\
798 \\
594\end{array}$ & $\begin{array}{l}1 \\
1 \\
- \\
- \\
-\end{array}$ & $\begin{array}{r}110 \\
12 \\
72 \\
20 \\
6\end{array}$ & $\begin{array}{r}18 \\
6 \\
1 \\
4 \\
7\end{array}$ & $\begin{array}{r}1,794 \\
1,286 \\
- \\
267 \\
241\end{array}$ \\
\hline $\begin{array}{l}\text { E.N. CENTRAL } \\
\text { Ohio } \\
\text { Ind. } \\
\text { III. } \\
\text { Mich. } \\
\text { Wis. }\end{array}$ & $\begin{array}{r}2,695 \\
1,083 \\
244 \\
792 \\
278 \\
298\end{array}$ & $\begin{array}{r}4,056 \\
1,140 \\
357 \\
1,542 \\
538 \\
479\end{array}$ & $\begin{array}{r}36 \\
8 \\
2 \\
12 \\
14 \\
-\end{array}$ & $\begin{array}{r}2,062 \\
334 \\
185 \\
1,053 \\
430 \\
60\end{array}$ & $\begin{array}{r}2,247 \\
303 \\
215 \\
1,189 \\
452 \\
88\end{array}$ & $\begin{array}{l}8 \\
1 \\
2 \\
3 \\
1 \\
1\end{array}$ & $\begin{array}{r}73 \\
7 \\
7 \\
46 \\
6 \\
7\end{array}$ & $\begin{array}{r}44 \\
27 \\
5 \\
10 \\
2 \\
-\end{array}$ & $\begin{array}{r}66 \\
4 \\
13 \\
19 \\
14 \\
16\end{array}$ \\
\hline $\begin{array}{l}\text { W.N. CENTRAL } \\
\text { Minn. } \\
\text { lowa } \\
\text { Mo. } \\
\text { N. Dak. } \\
\text { S. Dak. } \\
\text { Nebr. } \\
\text { Kans. }\end{array}$ & $\begin{array}{r}1,126 \\
49 \\
71 \\
942 \\
- \\
1 \\
11 \\
52\end{array}$ & $\begin{array}{r}1,576 \\
56 \\
64 \\
1,326 \\
4 \\
2 \\
10 \\
114\end{array}$ & $\begin{array}{r}26 \\
1 \\
8 \\
7 \\
1 \\
- \\
4 \\
5\end{array}$ & $\begin{array}{r}548 \\
126 \\
60 \\
232 \\
8 \\
25 \\
19 \\
78\end{array}$ & $\begin{array}{r}499 \\
73 \\
59 \\
243 \\
7 \\
14 \\
23 \\
80\end{array}$ & $\begin{array}{r}39 \\
1 \\
- \\
25 \\
1 \\
2 \\
3 \\
7\end{array}$ & $\begin{array}{l}1 \\
- \\
- \\
1 \\
- \\
- \\
-\end{array}$ & $\begin{array}{r}38 \\
- \\
1 \\
19 \\
- \\
13 \\
1 \\
4\end{array}$ & $\begin{array}{r}214 \\
17 \\
85 \\
26 \\
12 \\
39 \\
- \\
35\end{array}$ \\
\hline $\begin{array}{l}\text { S. ATLANTIC } \\
\text { Del. } \\
\text { Md. } \\
\text { D.C. } \\
\text { Va. } \\
\text { W. Va. } \\
\text { N.C. } \\
\text { S.C. } \\
\text { Ga. } \\
\text { Fla. }\end{array}$ & $\begin{array}{r}5,227 \\
25 \\
310 \\
210 \\
788 \\
9 \\
1,601 \\
775 \\
782 \\
727\end{array}$ & $\begin{array}{r}6,302 \\
91 \\
351 \\
323 \\
644 \\
12 \\
1,846 \\
895 \\
1,052 \\
1,088\end{array}$ & $\begin{array}{l}8 \\
- \\
- \\
- \\
1 \\
- \\
1 \\
- \\
1 \\
5\end{array}$ & $\begin{array}{r}3,889 \\
40 \\
322 \\
107 \\
292 \\
77 \\
516 \\
365 \\
665 \\
1,505\end{array}$ & $\begin{array}{r}4,379 \\
47 \\
382 \\
156 \\
415 \\
72 \\
565 \\
384 \\
731 \\
1,627\end{array}$ & $\begin{array}{l}2 \\
- \\
1 \\
- \\
- \\
- \\
- \\
1 \\
-\end{array}$ & $\begin{array}{r}48 \\
1 \\
14 \\
1 \\
8 \\
- \\
- \\
- \\
2\end{array}$ & $\begin{array}{r}207 \\
24 \\
- \\
19 \\
2 \\
82 \\
20 \\
55 \\
5\end{array}$ & $\begin{array}{r}1,928 \\
41 \\
502 \\
3 \\
418 \\
80 \\
166 \\
172 \\
359 \\
187\end{array}$ \\
\hline $\begin{array}{l}\text { E.S. CENTRAL } \\
\text { Ky. } \\
\text { Tenn. } \\
\text { Ala. } \\
\text { Miss. }\end{array}$ & $\begin{array}{r}3,779 \\
212 \\
967 \\
621 \\
1,979\end{array}$ & $\begin{array}{r}4,004 \\
330 \\
1,137 \\
852 \\
1,685\end{array}$ & $\begin{array}{l}6 \\
2 \\
3 \\
1 \\
-\end{array}$ & $\begin{array}{r}1,359 \\
315 \\
401 \\
421 \\
222\end{array}$ & $\begin{array}{r}1,565 \\
360 \\
489 \\
477 \\
239\end{array}$ & $\begin{array}{l}2 \\
2 \\
- \\
- \\
-\end{array}$ & $\begin{array}{l}3 \\
1 \\
2 \\
- \\
-\end{array}$ & $\begin{array}{r}45 \\
9 \\
29 \\
2 \\
5\end{array}$ & $\begin{array}{r}215 \\
25 \\
71 \\
119 \\
-\end{array}$ \\
\hline $\begin{array}{l}\text { W.S. CENTRAL } \\
\text { Ark. } \\
\text { La. } \\
\text { Okla. } \\
\text { Tex. }\end{array}$ & $\begin{array}{r}4,178 \\
462 \\
1,616 \\
111 \\
1,989\end{array}$ & $\begin{array}{r}5,294 \\
538 \\
2,479 \\
272 \\
2,005\end{array}$ & $\begin{array}{l}2 \\
- \\
- \\
2 \\
-\end{array}$ & $\begin{array}{r}2,882 \\
272 \\
193 \\
232 \\
2,185\end{array}$ & $\begin{array}{r}2,593 \\
185 \\
301 \\
164 \\
1,943\end{array}$ & $\begin{array}{r}17 \\
16 \\
- \\
1 \\
-\end{array}$ & $\begin{array}{r}16 \\
- \\
3 \\
3 \\
10\end{array}$ & $\begin{array}{r}53 \\
11 \\
- \\
35 \\
7\end{array}$ & $\begin{array}{r}643 \\
25 \\
69 \\
41 \\
508\end{array}$ \\
\hline $\begin{array}{l}\text { MOUNTAIN } \\
\text { Mont. } \\
\text { Idaho } \\
\text { Wyo. } \\
\text { Colo. } \\
\text { N. Mex. } \\
\text { Ariz. } \\
\text { Utah } \\
\text { Nev. }\end{array}$ & $\begin{array}{r}223 \\
4 \\
2 \\
2 \\
119 \\
19 \\
39 \\
8 \\
30\end{array}$ & $\begin{array}{r}240 \\
1 \\
- \\
8 \\
85 \\
24 \\
94 \\
11 \\
17\end{array}$ & $\begin{array}{r}13 \\
- \\
3 \\
- \\
6 \\
5 \\
2 \\
2 \\
-\end{array}$ & $\begin{array}{r}490 \\
9 \\
12 \\
9 \\
21 \\
65 \\
223 \\
51 \\
100\end{array}$ & $\begin{array}{r}533 \\
13 \\
12 \\
6 \\
79 \\
59 \\
235 \\
30 \\
99\end{array}$ & $\begin{array}{l}9 \\
3 \\
- \\
- \\
1 \\
1 \\
- \\
2 \\
2\end{array}$ & $\begin{array}{r}12 \\
- \\
- \\
- \\
3 \\
1 \\
3 \\
2 \\
3\end{array}$ & $\begin{array}{r}14 \\
4 \\
- \\
2 \\
4 \\
2 \\
1 \\
- \\
1\end{array}$ & $\begin{array}{r}135 \\
22 \\
3 \\
19 \\
15 \\
8 \\
45 \\
13 \\
10\end{array}$ \\
\hline $\begin{array}{l}\text { PACIFIC } \\
\text { Wash. } \\
\text { Oreg. } \\
\text { Calif. } \\
\text { Alaska } \\
\text { Hawaii }\end{array}$ & $\begin{array}{r}650 \\
32 \\
21 \\
590 \\
4 \\
3\end{array}$ & $\begin{array}{r}1,172 \\
55 \\
40 \\
1,061 \\
8 \\
8\end{array}$ & $\begin{array}{r}53 \\
3 \\
- \\
46 \\
- \\
4\end{array}$ & $\begin{array}{r}5,176 \\
250 \\
90 \\
4,531 \\
63 \\
242\end{array}$ & $\begin{array}{r}4,941 \\
260 \\
- \\
4,385 \\
56 \\
240\end{array}$ & $\begin{array}{l}5 \\
- \\
2 \\
2 \\
1 \\
-\end{array}$ & $\begin{array}{r}111 \\
4 \\
5 \\
97 \\
- \\
5\end{array}$ & $\begin{array}{l}- \\
- \\
- \\
- \\
- \\
-\end{array}$ & $\begin{array}{r}273 \\
- \\
12 \\
231 \\
30 \\
-\end{array}$ \\
\hline $\begin{array}{l}\text { Guam } \\
\text { P.R. } \\
\text { V.I. } \\
\text { Amer. Samoa } \\
\text { C.N.M.I. }\end{array}$ & $\begin{array}{r}10 \\
287 \\
28 \\
1 \\
2\end{array}$ & $\begin{array}{r}3 \\
479 \\
41 \\
- \\
7\end{array}$ & $\begin{array}{l}- \\
- \\
- \\
-\end{array}$ & $\begin{array}{r}170 \\
159 \\
- \\
4 \\
35\end{array}$ & $\begin{array}{r}65 \\
213 \\
2 \\
4 \\
41\end{array}$ & $\begin{array}{l}- \\
- \\
- \\
-\end{array}$ & $\begin{array}{l}1 \\
- \\
1 \\
1\end{array}$ & $\begin{array}{l}- \\
- \\
- \\
-\end{array}$ & $\begin{array}{r}- \\
60 \\
- \\
-\end{array}$ \\
\hline
\end{tabular}


TABLE III. Deaths in 121 U.S. cities, ${ }^{*}$ week ending December 17, 1994 (50th Week)

\begin{tabular}{|c|c|c|c|c|c|c|c|c|c|c|c|c|c|c|c|}
\hline \multirow[b]{2}{*}{ Reporting Area } & \multicolumn{6}{|c|}{ All Causes, By Age (Years) } & \multirow{2}{*}{$\begin{array}{l}\text { P\&l }^{\dagger} \\
\text { Total }\end{array}$} & \multirow[b]{2}{*}{ Reporting Area } & \multicolumn{6}{|c|}{ All Causes, By Age (Years) } & \multirow{2}{*}{ Total $^{\text {P\& }}$} \\
\hline & $\begin{array}{c}\text { All } \\
\text { Ages }\end{array}$ & $\geq 65$ & 45-64 & 25-44 & $1-24$ & $<1$ & & & $\begin{array}{c}\text { All } \\
\text { Ages }\end{array}$ & $\geq 65$ & 45-64 & $25-44$ & $1-24$ & $<1$ & \\
\hline $\begin{array}{l}\text { NEW ENGLAND } \\
\text { Boston, Mass. } \\
\text { Bridgeport, Conn. } \\
\text { Cambridge, Mass. } \\
\text { Fall River, Mass. } \\
\text { Hartford, Conn. } \\
\text { Lowell, Mass. } \\
\text { Lynn, Mass. } \\
\text { New Bedford, Mass. } \\
\text { New Haven, Conn. } \\
\text { Providence, R.I. } \\
\text { Somerville, Mass. } \\
\text { Springfield, Mass. } \\
\text { Waterbury, Conn. } \\
\text { Worcester, Mass. }\end{array}$ & $\begin{array}{r}631 \\
172 \\
49 \\
26 \\
36 \\
56 \\
17 \\
21 \\
28 \\
41 \\
57 \\
10 \\
42 \\
23 \\
53\end{array}$ & $\begin{array}{r}448 \\
110 \\
36 \\
19 \\
30 \\
37 \\
12 \\
18 \\
24 \\
27 \\
44 \\
6 \\
27 \\
16 \\
42\end{array}$ & $\begin{array}{r}103 \\
34 \\
5 \\
6 \\
4 \\
10 \\
5 \\
2 \\
4 \\
7 \\
5 \\
3 \\
9 \\
4 \\
5\end{array}$ & $\begin{array}{r}50 \\
18 \\
4 \\
1 \\
2 \\
5 \\
- \\
- \\
- \\
6 \\
5 \\
1 \\
3 \\
2 \\
3\end{array}$ & $\begin{array}{r}17 \\
7 \\
2 \\
- \\
- \\
3 \\
- \\
- \\
- \\
- \\
3 \\
- \\
1 \\
1 \\
-\end{array}$ & $\begin{array}{r}13 \\
3 \\
2 \\
- \\
- \\
1 \\
- \\
1 \\
- \\
1 \\
- \\
- \\
2 \\
- \\
3\end{array}$ & $\begin{array}{r}34 \\
14 \\
1 \\
2 \\
- \\
- \\
1 \\
1 \\
- \\
2 \\
4 \\
- \\
1 \\
4 \\
4\end{array}$ & $\begin{array}{l}\text { S. ATLANTIC } \\
\text { Atlanta, Ga. } \\
\text { Baltimore, Md. } \\
\text { Charlotte, N.C. } \\
\text { Jacksonville, Fla. } \\
\text { Miami, Fla. } \\
\text { Norfolk, Va. } \\
\text { Richmond, Va. } \\
\text { Savannah, Ga. } \\
\text { St. Petersburg, Fla. } \\
\text { Tampa, Fla. } \\
\text { Washington, D.C. } \\
\text { Wilmington, Del. } \\
\text { E.S. CENTRAL } \\
\text { Birminaham. Ala }\end{array}$ & $\begin{array}{r}1,453 \\
188 \\
295 \\
119 \\
132 \\
100 \\
39 \\
101 \\
50 \\
60 \\
176 \\
182 \\
11 \\
914 \\
131\end{array}$ & $\begin{array}{r}912 \\
109 \\
183 \\
72 \\
92 \\
64 \\
27 \\
56 \\
36 \\
50 \\
117 \\
99 \\
7 \\
551 \\
63\end{array}$ & $\begin{array}{r}247 \\
31 \\
51 \\
24 \\
18 \\
14 \\
7 \\
22 \\
9 \\
6 \\
23 \\
40 \\
2 \\
176 \\
28\end{array}$ & $\begin{array}{r}214 \\
31 \\
49 \\
15 \\
13 \\
17 \\
2 \\
17 \\
4 \\
4 \\
27 \\
34 \\
1 \\
103 \\
21\end{array}$ & $\begin{array}{r}42 \\
8 \\
6 \\
4 \\
3 \\
2 \\
1 \\
4 \\
1 \\
- \\
7 \\
6 \\
- \\
47 \\
9\end{array}$ & $\begin{array}{r}36 \\
9 \\
6 \\
4 \\
5 \\
3 \\
2 \\
2 \\
- \\
- \\
2 \\
3 \\
- \\
37 \\
10\end{array}$ & $\begin{array}{r}71 \\
3 \\
28 \\
4 \\
11 \\
- \\
1 \\
5 \\
3 \\
4 \\
8 \\
4 \\
- \\
58 \\
5\end{array}$ \\
\hline $\begin{array}{l}\text { MID. ATLANTIC } \\
\text { Albany, N.Y. } \\
\text { Allentown, Pa. } \\
\text { Buffalo, N.Y. } \\
\text { Camden, N.J. } \\
\text { Elizabeth, N.J. } \\
\text { Erie, Pa.§ }\end{array}$ & $\begin{array}{r}2,806 \\
56 \\
33 \\
95 \\
45 \\
26 \\
42\end{array}$ & $\begin{array}{r}1,853 \\
38 \\
23 \\
78 \\
29 \\
21 \\
30\end{array}$ & $\begin{array}{r}556 \\
11 \\
5 \\
10 \\
7 \\
3 \\
10\end{array}$ & $\begin{array}{r}283 \\
2 \\
4 \\
5 \\
4 \\
2 \\
1\end{array}$ & $\begin{array}{r}59 \\
2 \\
1 \\
- \\
2 \\
- \\
1\end{array}$ & $\begin{array}{r}55 \\
3 \\
- \\
2 \\
3 \\
- \\
-\end{array}$ & $\begin{array}{r}162 \\
3 \\
1 \\
28 \\
3 \\
4 \\
4\end{array}$ & $\begin{array}{l}\text { Chattanooga, Tenn. } \\
\text { Knoxville, Tenn. } \\
\text { Lexington, Ky. } \\
\text { Memphis, Tenn. } \\
\text { Mobile, Ala. } \\
\text { Montgomery, Ala. } \\
\text { Nashville, Tenn. }\end{array}$ & $\begin{array}{r}70 \\
109 \\
62 \\
243 \\
109 \\
55 \\
135\end{array}$ & $\begin{array}{r}47 \\
74 \\
37 \\
160 \\
62 \\
36 \\
72\end{array}$ & $\begin{array}{l}20 \\
14 \\
20 \\
14 \\
45 \\
22 \\
10 \\
23\end{array}$ & $\begin{array}{r}4 \\
12 \\
8 \\
14 \\
14 \\
5 \\
25\end{array}$ & $\begin{array}{r}2 \\
3 \\
2 \\
2 \\
10 \\
7 \\
2 \\
12\end{array}$ & $\begin{array}{r}10 \\
2 \\
1 \\
1 \\
14 \\
4 \\
2 \\
3\end{array}$ & $\begin{array}{r}1 \\
14 \\
6 \\
6 \\
17 \\
3 \\
2 \\
10\end{array}$ \\
\hline $\begin{array}{l}\text { Jersey City, N.J. } \\
\text { New York City, N.Y. } \\
\text { Newark, N.J. } \\
\text { Paterson, N.J. } \\
\text { Philadelphia, Pa. } \\
\text { Pittsburgh, Pa.§ } \\
\text { Reading, Pa. } \\
\text { Rochester, N.Y. } \\
\text { Schenectady, N.Y. } \\
\text { Scranton, Pa.§ } \\
\text { Syracuse, N.Y. } \\
\text { Trenton, N.J. } \\
\text { Utica, N.Y. } \\
\text { Yonkers, N.Y. }\end{array}$ & $\begin{array}{r}52 \\
1,516 \\
62 \\
34 \\
400 \\
99 \\
17 \\
126 \\
21 \\
27 \\
104 \\
37 \\
14 \\
U\end{array}$ & $\begin{array}{r}28 \\
949 \\
29 \\
25 \\
272 \\
72 \\
11 \\
96 \\
18 \\
23 \\
78 \\
21 \\
12 \\
U\end{array}$ & $\begin{array}{r}13 \\
322 \\
20 \\
3 \\
66 \\
19 \\
4 \\
25 \\
3 \\
4 \\
20 \\
10 \\
1 \\
U\end{array}$ & $\begin{array}{r}7 \\
192 \\
10 \\
5 \\
37 \\
4 \\
2 \\
1 \\
- \\
- \\
4 \\
2 \\
1 \\
\text { U }\end{array}$ & $\begin{array}{r}2 \\
24 \\
3 \\
1 \\
16 \\
2 \\
- \\
2 \\
- \\
- \\
1 \\
2 \\
- \\
\end{array}$ & $\begin{array}{r}2 \\
29 \\
- \\
- \\
9 \\
2 \\
- \\
2 \\
- \\
- \\
1 \\
2 \\
- \\
\text { U }\end{array}$ & $\begin{array}{r}4 \\
50 \\
1 \\
1 \\
24 \\
11 \\
6 \\
11 \\
3 \\
2 \\
3 \\
3 \\
-\end{array}$ & $\begin{array}{l}\text { W.S. CENTRAL } \\
\text { Austin, Tex. } \\
\text { Baton Rouge, La. } \\
\text { Corpus Christi, Tex. } \\
\text { Dallas, Tex. } \\
\text { El Paso, Tex. } \\
\text { Ft. Worth, Tex. } \\
\text { Houston, Tex. } \\
\text { Little Rock, Ark. } \\
\text { New Orleans, La. } \\
\text { San Antonio, Tex. } \\
\text { Shreveport, La. } \\
\text { Tulsa, Okla. }\end{array}$ & $\begin{array}{r}1,498 \\
82 \\
25 \\
U \\
237 \\
57 \\
116 \\
390 \\
71 \\
107 \\
245 \\
54 \\
114\end{array}$ & $\begin{array}{r}948 \\
52 \\
20 \\
U \\
142 \\
39 \\
74 \\
219 \\
45 \\
67 \\
167 \\
36 \\
87\end{array}$ & $\begin{array}{r}297 \\
12 \\
3 \\
\cup \\
50 \\
13 \\
22 \\
90 \\
13 \\
20 \\
49 \\
11 \\
14\end{array}$ & $\begin{array}{r}176 \\
16 \\
2 \\
U \\
34 \\
4 \\
10 \\
59 \\
7 \\
12 \\
21 \\
4 \\
7\end{array}$ & $\begin{array}{r}40 \\
1 \\
- \\
\cup \\
4 \\
- \\
5 \\
9 \\
6 \\
7 \\
3 \\
1 \\
4\end{array}$ & $\begin{array}{r}37 \\
1 \\
- \\
U \\
7 \\
1 \\
5 \\
13 \\
- \\
1 \\
5 \\
2 \\
2\end{array}$ & $\begin{array}{r}94 \\
3 \\
3 \\
U \\
5 \\
5 \\
4 \\
37 \\
9 \\
- \\
21 \\
3 \\
4\end{array}$ \\
\hline $\begin{array}{l}\text { E.N. CENTRAL } \\
\text { Akron, Ohio } \\
\text { Canton, Ohio } \\
\text { Chicago, III. } \\
\text { Cincinnati, Ohio } \\
\text { Cleveland, Ohio } \\
\text { Columbus, Ohio } \\
\text { Dayton, Ohio } \\
\text { Detroit, Mich. } \\
\text { Evansville, Ind. }\end{array}$ & $\begin{array}{r}2,330 \\
46 \\
36 \\
449 \\
205 \\
170 \\
167 \\
115 \\
252 \\
44\end{array}$ & $\begin{array}{r}1,480 \\
38 \\
25 \\
188 \\
136 \\
106 \\
105 \\
86 \\
167 \\
36\end{array}$ & $\begin{array}{r}423 \\
5 \\
10 \\
78 \\
46 \\
41 \\
37 \\
16 \\
41 \\
6\end{array}$ & $\begin{array}{r}228 \\
1 \\
- \\
103 \\
12 \\
12 \\
14 \\
7 \\
22 \\
2\end{array}$ & $\begin{array}{r}115 \\
1 \\
- \\
66 \\
6 \\
3 \\
1 \\
3 \\
7 \\
-\end{array}$ & $\begin{array}{r}83 \\
1 \\
1 \\
14 \\
5 \\
8 \\
10 \\
3 \\
15 \\
-\end{array}$ & $\begin{array}{r}133 \\
- \\
3 \\
22 \\
19 \\
5 \\
9 \\
9 \\
7 \\
4\end{array}$ & $\begin{array}{l}\text { MOUNTAIN } \\
\text { Albuquerque, N.M. } \\
\text { Colo. Springs, Colo. } \\
\text { Denver, Colo. } \\
\text { Las Vegas, Nev. } \\
\text { Ogden, Utah } \\
\text { Phoenix, Ariz. } \\
\text { Pueblo, Colo. } \\
\text { Salt Lake City, Utah } \\
\text { Tucson, Ariz. }\end{array}$ & $\begin{array}{r}906 \\
105 \\
57 \\
98 \\
133 \\
24 \\
204 \\
30 \\
124 \\
131\end{array}$ & $\begin{array}{r}597 \\
67 \\
41 \\
59 \\
85 \\
21 \\
119 \\
20 \\
83 \\
102\end{array}$ & $\begin{array}{r}190 \\
22 \\
13 \\
23 \\
28 \\
2 \\
44 \\
9 \\
28 \\
21\end{array}$ & $\begin{array}{r}75 \\
10 \\
2 \\
10 \\
16 \\
- \\
27 \\
1 \\
6 \\
3\end{array}$ & $\begin{array}{r}28 \\
5 \\
- \\
1 \\
4 \\
- \\
10 \\
- \\
6 \\
2\end{array}$ & $\begin{array}{r}16 \\
1 \\
1 \\
5 \\
- \\
1 \\
4 \\
- \\
1 \\
3\end{array}$ & $\begin{array}{r}71 \\
5 \\
4 \\
2 \\
7 \\
4 \\
22 \\
3 \\
14 \\
10\end{array}$ \\
\hline $\begin{array}{l}\text { Fort Wayne, Ind. } \\
\text { Gary, Ind. } \\
\text { Grand Rapids, Mich. } \\
\text { Indianapolis, Ind. } \\
\text { Madison, Wis. } \\
\text { Milwaukee, Wis. } \\
\text { Peoria, III. } \\
\text { Rockford, III. } \\
\text { South Bend, Ind. } \\
\text { Toledo, Ohio } \\
\text { Youngstown, Ohio }\end{array}$ & $\begin{array}{r}71 \\
20 \\
51 \\
213 \\
45 \\
120 \\
33 \\
45 \\
41 \\
136 \\
71\end{array}$ & $\begin{array}{r}48 \\
10 \\
42 \\
127 \\
37 \\
82 \\
28 \\
29 \\
30 \\
103 \\
57\end{array}$ & $\begin{array}{r}14 \\
4 \\
6 \\
43 \\
3 \\
20 \\
2 \\
11 \\
8 \\
23 \\
9\end{array}$ & $\begin{array}{r}3 \\
2 \\
2 \\
20 \\
4 \\
9 \\
2 \\
2 \\
3 \\
7 \\
1\end{array}$ & $\begin{array}{l}5 \\
2 \\
1 \\
9 \\
1 \\
3 \\
- \\
2 \\
- \\
3 \\
2\end{array}$ & $\begin{array}{r}1 \\
1 \\
- \\
14 \\
- \\
6 \\
1 \\
1 \\
- \\
- \\
2\end{array}$ & $\begin{array}{r}4 \\
- \\
5 \\
16 \\
5 \\
7 \\
3 \\
4 \\
1 \\
6 \\
4\end{array}$ & $\begin{array}{l}\text { PACIFIC } \\
\text { Berkeley, Calif. } \\
\text { Fresno, Calif. } \\
\text { Glendale, Calif. } \\
\text { Honolulu, Hawaii } \\
\text { Long Beach, Calif. } \\
\text { Los Angeles, Calif. } \\
\text { Pasadena, Calif. } \\
\text { Portland, Oreg. } \\
\text { Sacramento, Calif. } \\
\text { San Diego, Calif. }\end{array}$ & $\begin{array}{r}1,332 \\
14 \\
100 \\
U \\
80 \\
85 \\
U \\
34 \\
132 \\
U \\
192\end{array}$ & $\begin{array}{r}942 \\
11 \\
71 \\
U \\
57 \\
62 \\
U \\
24 \\
100 \\
U \\
128\end{array}$ & $\begin{array}{r}218 \\
1 \\
18 \\
U \\
16 \\
10 \\
U \\
6 \\
17 \\
U \\
36\end{array}$ & $\begin{array}{r}101 \\
2 \\
6 \\
U \\
3 \\
9 \\
U \\
3 \\
11 \\
U \\
9\end{array}$ & $\begin{array}{r}19 \\
- \\
4 \\
U \\
1 \\
1 \\
U \\
- \\
2 \\
U \\
4\end{array}$ & $\begin{array}{r}39 \\
- \\
1 \\
U \\
3 \\
3 \\
U \\
1 \\
2 \\
U \\
15\end{array}$ & $\begin{array}{r}125 \\
- \\
10 \\
U \\
3 \\
14 \\
U \\
1 \\
8 \\
U \\
26\end{array}$ \\
\hline $\begin{array}{l}\text { W.N. CENTRAL } \\
\text { Des Moines, lowa } \\
\text { Duluth, Minn. } \\
\text { Kansas City, Kans. } \\
\text { Kansas City, Mo. } \\
\text { Lincoln, Nebr. }\end{array}$ & $\begin{array}{r}853 \\
203 \\
10 \\
19 \\
122 \\
30\end{array}$ & $\begin{array}{r}603 \\
148 \\
9 \\
12 \\
71 \\
25\end{array}$ & $\begin{array}{r}141 \\
33 \\
- \\
2 \\
24 \\
2\end{array}$ & $\begin{array}{r}61 \\
13 \\
- \\
5 \\
11 \\
2\end{array}$ & $\begin{array}{r}19 \\
5 \\
1 \\
- \\
3 \\
-\end{array}$ & $\begin{array}{r}17 \\
3 \\
- \\
- \\
2 \\
1\end{array}$ & $\begin{array}{r}62 \\
22 \\
- \\
1 \\
11 \\
2\end{array}$ & $\begin{array}{l}\text { San Francisco, Calif. } \\
\text { San Jose, Calif. } \\
\text { Santa Cruz, Calif. } \\
\text { Seattle, Wash. } \\
\text { Spokane, Wash. } \\
\text { Tacoma, Wash. }\end{array}$ & $\begin{array}{r}133 \\
247 \\
25 \\
150 \\
59 \\
81\end{array}$ & $\begin{array}{r}75 \\
180 \\
18 \\
111 \\
40 \\
65\end{array}$ & $\begin{array}{r}23 \\
38 \\
4 \\
24 \\
12 \\
13\end{array}$ & $\begin{array}{r}18 \\
22 \\
2 \\
9 \\
4 \\
3\end{array}$ & $\begin{array}{l}1 \\
2 \\
- \\
3 \\
1 \\
-\end{array}$ & $\begin{array}{l}3 \\
5 \\
1 \\
3 \\
2 \\
-\end{array}$ & $\begin{array}{r}20 \\
29 \\
3 \\
5 \\
1 \\
5\end{array}$ \\
\hline $\begin{array}{l}\text { Minneapolis, Minn. } \\
\text { Omaha, Nebr. } \\
\text { St. Louis, Mo. } \\
\text { St. Paul, Minn. } \\
\text { Wichita, Kans. }\end{array}$ & $\begin{array}{r}162 \\
70 \\
122 \\
57 \\
58\end{array}$ & $\begin{array}{r}118 \\
48 \\
85 \\
43 \\
44\end{array}$ & $\begin{array}{r}27 \\
13 \\
23 \\
10 \\
7\end{array}$ & $\begin{array}{r}12 \\
7 \\
5 \\
2 \\
4\end{array}$ & $\begin{array}{l}1 \\
- \\
6 \\
- \\
3\end{array}$ & $\begin{array}{l}4 \\
2 \\
3 \\
2 \\
-\end{array}$ & $\begin{array}{r}7 \\
4 \\
11 \\
4 \\
-\end{array}$ & TOTAL & $12,723^{\mathbb{I}}$ & 8,334 & 2,351 & 1,291 & 386 & 333 & 810 \\
\hline
\end{tabular}

* Mortality data in this table are voluntarily reported from 121 cities in the United States, most of which have populations of 100,000 or more. A death is reported by the place of its occurrence and by the week that the death certificate was filed. Fetal deaths are not included.

†Pneumonia and influenza.

${ }^{\S}$ Because of changes in reporting methods in these 3 Pennsylvania cities, these numbers are partial counts for the current week. Complete counts will be available in 4 to 6 weeks.

ITotal includes unknown ages.

U: Unavailable. 


\section{Abortion Surveillance - Continued}

References

1. CDC. Abortion surveillance: preliminary data-United States, 1991. MMWR 1994;43:42-4.

2. NCHS. Advance report of final natality statistics, 1992. Hyattsville, Maryland: US Department of Health and Human Services, Public Health Service, CDC, 1994. (Monthly vital statistics report; vol 43 , no. 5 , suppl).

3. CDC. Abortion surveillance, 1977. Atlanta: US Department of Health and Human Services, Public Health Service, 1979.

\section{Current Trends}

\section{State-Specific Trends Among Women Who Did Not Receive Prenatal Care — United States, 1980-1992}

Lack of prenatal care is strongly associated with an increased risk for low birthweight ( $<2500 \mathrm{~g}[<5 \mathrm{lbs} 8 \mathrm{oz}]$ at birth) infants, preterm delivery, and maternal and infant mortality (1). From 1980 through 1992, the nationally aggregated percentage of pregnant women who did not receive prenatal care increased by $31 \%$-from $1.3 \%$ to $1.7 \%(2,3)$. Because nationally aggregated data can obscure variations among states, CDC analyzed state-specific data derived from birth certificates for 1980-1992 to examine trends among women who did not receive prenatal care. This report summarizes the findings of the analysis.

Prenatal-care data were ascertained from the section on the birth certificate indicating the month of pregnancy in which prenatal care was initiated. State-specific percentages for each year from 1980 through 1992 were ordered from lowest to highest to determine the 25th, 50th (i.e., median), and 75th percentiles and maximum value for each year. In addition, state-specific percentages for women who did not receive prenatal care were compared for 1980-1981 and 1991-1992 using the total number of births to women who did not receive prenatal care and the total number of births. Absolute change was calculated by comparing the percentages for 1980-1981 with those for 1991-1992.

From 1980 to 1989 , the median state-specific percentage of births to women who did not receive prenatal care increased from $0.8 \%$ to $1.3 \%$ (Figure 1). Although patterns for the 25th and 75th percentiles were similar, the maximum value increased substantially-from $3.7 \%$ in 1980 to $7.5 \%$ in 1989; in 1992, the percentage declined to $4.8 \%$. Percentages were consistently high in the District of Columbia, Florida, New Mexico, New York, and Texas.

For 1980-1981, the percentage of women who did not receive prenatal care ranged from $0.14 \%$ (Vermont) to $3.67 \%$ (New York) (Table 1); for 1991-1992, the percentages ranged from $0.32 \%$ (Utah) to $5.63 \%$ (District of Columbia). When compared with 1980 1981, during 1991-1992 the percentage of women who did not receive prenatal care declined in eight states (Florida, Kentucky, New Jersey, New York, Oklahoma, Rhode Island, South Dakota, and Utah) and increased in 42 states and the District of Columbia; in nine states, the increase was greater than $100 \%$ (Delaware, Illinois, Indiana, Louisiana, Michigan, Ohio, Pennsylvania, Vermont, and Wisconsin).

Reported by: Div of Reproductive Health, National Center for Chronic Disease Prevention and Health Promotion; Div of Health and Utilization Analysis, National Center for Health Statistics, $C D C$. 
Prenatal Care - Continued

FIGURE 1. State-specific percentages* of women who did not receive prenatal care, by percentile and year - United States, 1980-1992

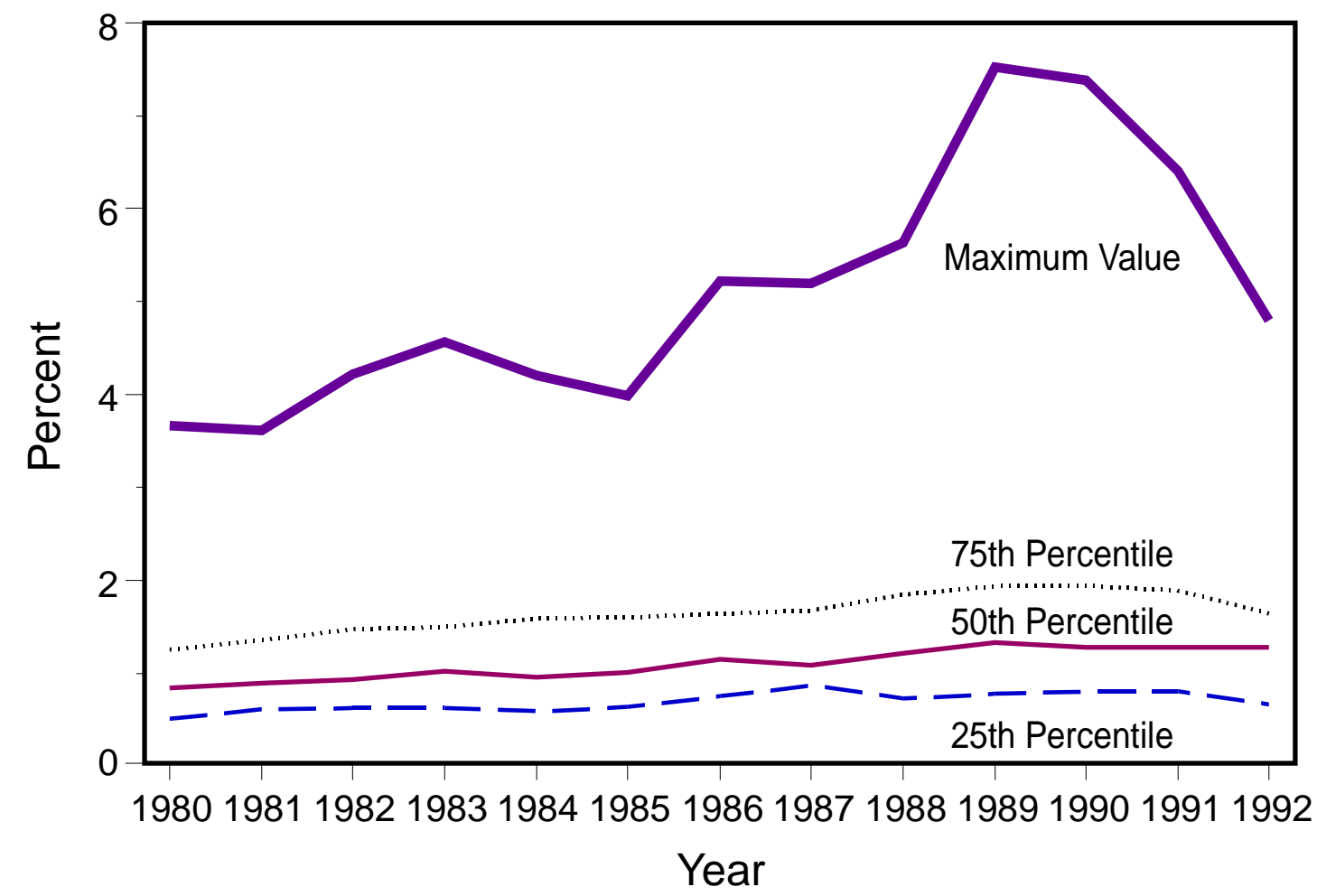

* Data from the 50 states and the District of Columbia were ranked. Observations 13 (25th percentile), 26 (50th percentile), 39 (75th percentile) and 51 (maximum value) were plotted for each year.

Editorial Note: Federal and state initiatives during the mid-1980s aimed to increase access to prenatal care by expanding Medicaid eligibility and increasing funding for maternal and child health block grants and other state-funded programs $(4,5)$. The decrease in the percentage of births to women who did not receive prenatal care during 1991-1992 is the most substantial decrease recorded since 1969 (the first year data about prenatal-care initiation were reported). Despite these improvements, approximately 69,000 women did not receive prenatal care in 1992.

Health agencies commonly use aggregated data to describe national patterns in prenatal care in the United States. However, the findings in this and other reports indicate the importance of using state-specific data to fully elucidate and better understand long-term trends $(6)$. In particular, these findings documented substantial variation among states for the percentage of women who did not receive prenatal care. For example, the comparison of data for 1980-1981 with 1991-1992 demonstrated slight decreases in the percentage of women who did not receive prenatal care in eight states and substantial increases in nine others.

The findings in this report are subject to at least two limitations. First, the overall increase in the percentage of women who did not receive prenatal care may have been related to improved case ascertainment. From 1980 to 1992, the percentage of 


\section{Prenatal Care - Continued}

TABLE 1. Percentages of women who did not receive prenatal care, by state - United States, 1980-1981 and 1991-1992

\begin{tabular}{|c|c|c|c|c|}
\hline State & 1980-1981 & 1991-1992 & $\begin{array}{c}\text { Absolute change* } \\
\text { from } 1980-1981 \\
\text { to } 1991-1992\end{array}$ & $\begin{array}{c}\% \text { Change* } \\
\text { from } 1980-1981 \\
\text { to } 1991-1992\end{array}$ \\
\hline Alabama & 1.36 & 1.38 & 0.03 & 1.86 \\
\hline Alaska & 0.68 & 0.80 & 0.12 & 17.60 \\
\hline Arizona & 1.93 & 2.24 & 0.30 & 15.74 \\
\hline Arkansas & 1.45 & 1.79 & 0.34 & 23.52 \\
\hline California & 0.93 & 1.33 & 0.40 & 43.61 \\
\hline Colorado & 0.79 & 1.03 & 0.25 & 31.53 \\
\hline Connecticut & 0.47 & 0.50 & 0.03 & 5.47 \\
\hline Delaware & 0.59 & 1.71 & 1.12 & 189.85 \\
\hline District of Columbia & 3.10 & 5.63 & 2.53 & 81.75 \\
\hline Florida & 2.05 & 1.85 & -0.20 & -9.90 \\
\hline Georgia & 1.34 & 2.13 & 0.79 & 58.84 \\
\hline Hawaii & 0.54 & 0.84 & 0.30 & 55.47 \\
\hline Idaho & 0.81 & 1.02 & 0.21 & 25.80 \\
\hline Illinois & 1.01 & 2.04 & 1.03 & 102.48 \\
\hline Indiana & 0.92 & 2.21 & 1.28 & 138.82 \\
\hline lowa & 0.36 & 0.53 & 0.17 & 47.52 \\
\hline Kansas & 0.51 & 0.76 & 0.25 & 48.00 \\
\hline Kentucky & 1.94 & 1.55 & -0.39 & -19.97 \\
\hline Louisiana & 1.21 & 2.53 & 1.33 & 109.92 \\
\hline Maine & 0.82 & 1.06 & 0.24 & 29.69 \\
\hline Maryland & 0.85 & 1.29 & 0.44 & 51.29 \\
\hline Massachusetts & 0.39 & 0.50 & 0.11 & 28.72 \\
\hline Michigan & 0.66 & 1.50 & 0.84 & 128.02 \\
\hline Mississippi & 0.42 & 0.49 & 0.07 & 17.07 \\
\hline Minnesota & 0.83 & 1.26 & 0.43 & 51.78 \\
\hline Missouri & 0.92 & 1.72 & 0.79 & 85.82 \\
\hline Montana & 0.74 & 0.87 & 0.13 & 18.18 \\
\hline Nebraska & 0.41 & 0.60 & 0.19 & 45.33 \\
\hline Nevada & 1.61 & 2.87 & 1.25 & 77.52 \\
\hline New Hampshire & 0.42 & 0.79 & 0.37 & 87.83 \\
\hline New Jersey & 1.63 & 1.25 & -0.38 & -23.38 \\
\hline New Mexico & 1.73 & 2.88 & 1.15 & 66.68 \\
\hline New York & 3.67 & 3.13 & -0.54 & -14.69 \\
\hline North Carolina & 0.90 & 1.69 & 0.80 & 88.60 \\
\hline North Dakota & 0.50 & 0.64 & 0.13 & 26.92 \\
\hline Ohio & 0.69 & 1.45 & 0.76 & 109.77 \\
\hline Oklahoma & 1.88 & 1.35 & -0.54 & -28.54 \\
\hline Oregon & 0.69 & 0.97 & 0.28 & 40.97 \\
\hline Pennsylvania & 0.68 & 1.80 & 1.12 & 165.33 \\
\hline Rhode Island & 0.47 & 0.40 & -0.07 & -14.27 \\
\hline South Carolina & 1.15 & 2.04 & 0.89 & 76.79 \\
\hline South Dakota & 2.43 & 1.16 & -1.28 & -52.43 \\
\hline Tennessee & 1.30 & 1.53 & 0.24 & 18.14 \\
\hline Texas & 2.71 & 3.82 & 1.11 & 41.09 \\
\hline Utah & 0.40 & 0.32 & -0.09 & -21.68 \\
\hline Vermont & 0.14 & 0.38 & 0.24 & 173.66 \\
\hline Virginia & 0.76 & 1.45 & 0.69 & 90.52 \\
\hline Washington & 0.58 & 0.83 & 0.25 & 42.87 \\
\hline West Virginia & 0.93 & 1.14 & 0.21 & 22.78 \\
\hline Wisconsin & 0.37 & 0.76 & 0.39 & 103.86 \\
\hline Wyoming & 0.79 & 0.88 & 0.09 & 11.71 \\
\hline
\end{tabular}




\section{Prenatal Care - Continued}

women for whom initiation of prenatal care was unknown decreased from $2.8 \%$ in 1980 to $2.2 \%$ in 1992 . However, the increase in the percentage of women who did not receive prenatal care may have been related to the decrease in the percentage of women who initiated prenatal care during the second trimester (from 18.1\% in 1980 to $16.7 \%$ in 1992). The percentages of women who initiated prenatal care during the first or third trimester remained unchanged $(74 \%$ and $4 \%$, respectively). Second, estimates of the prevalence of nonreceipt of prenatal care may be inaccurate because a standard method of measuring initiation of prenatal care is not available. Although maternal postpartum interview data (7) and birth certificate data identify similar percentages of women who do not receive prenatal care, these sources may not identify the same women. For example, in a national sample of women who gave birth in 1988, among those who were identified either by the birth certificate or maternal interview as not receiving prenatal care, only $33 \%$ were identified by both sources (7).

Because the importance of prenatal care is widely accepted and efforts are made to provide such care to all women, nonreceipt of prenatal care should be considered a sentinel health event. A sample of these episodes should be investigated to identify and implement interventions. In particular, public health workers need to determine the reasons for nonreceipt of prenatal care (e.g., choosing not to obtain care, inability to pay for care, or lack of providers or transportation), and state health departments should consider the financial, programmatic, and social factors that are associated with non receipt of prenatal care.

\section{References}

1. Office of Technology Assessment, US Congress. Healthy children: investing in the future. Washington, DC: US Congress, Office of Technology Assessment, 1988.

2. NCHS. Vital statistics of the United States, 1980. Vol I, natality. Washington, DC: US Department of Health and Human Services, Public Health Service, 1984; DHHS publication no. (PHS)851100.

3. Ventura SJ, Martin JA, Taffel SM, et al. Advance report of final natality statistics, 1992. Hyattsville, Maryland: US Department of Health and Human Services, Public Health Service, CDC, 1994. (Monthly vital statistics report; vol 43, no. 5, suppl).

4. Singh S, Forrest JD, Torres A. Prenatal care in the United States: a state and county inventory. Vols 1 and 2. New York: Alan Guttmacher Institute, 1989.

5. Committee to Study Outreach for Prenatal Care, National Institute of Medicine. Prenatal care: reaching mothers, reaching infants. Washington, DC: National Academy Press, 1988.

6. Ingram DD, Makuc D, Kleinman JC. National and state trends in use of prenatal care, 1970-83. Am J Public Health 1986;76:415-23.

7. Schoendorf KC, Parker JD, Batkhan LZ, Kiely JL. Comparability of the birth certificate and 1988 Maternal and Infant Health Survey. Hyattsville, Maryland: US Department of Health and Human Services, Public Health Service, CDC, NCHS, 1993; DHHS publication no. (PHS)93-1390. (Vital and health statistics; series 2, no. 116). 
International Notes

\section{Bolivian Hemorrhagic Fever - El Beni Department, Bolivia, 1994}

In July 1994, an outbreak of Bolivian hemorrhagic fever (BHF), which is caused by Machupo virus, began in northeastern Bolivia. This report describes the investigation and features of this outbreak, the search for additional cases of BHF in El Beni, Bolivia, and results of rodent investigations.

\section{Initial Investigation}

The outbreak initially occurred among members of an extended family residing in Magdalena (1994 population: approximately 5300) located in the north central Province of Iténez, El Beni Department (Figure 1). From July 4 through August 12, 1994, seven family members (aged 10 months-50 years) developed an illness characterized by fever, hypotension, subconjunctival and gingival bleeding, epistaxis, petechiae, tremor, and dysarthria. Six of these persons died; the person who had the index case survived. Laboratory studies performed on serum and tissue specimens from decedents confirmed the diagnosis of BHF by isolation of Machupo virus and detection of viral antigen in all five patients for whom specimens were available; the survivor developed enzyme-linked immunosorbent assay (ELISA) immunoglobulin $\mathrm{M}(\operatorname{IgM})$ and immunoglobulin G (IgG) antibodies to Machupo virus.

FIGURE 1. Area in which Bolivian hemorrhagic fever is endemic and four cities* in which suspected cases were identified - Bolivia, July 1-September 30, 1994

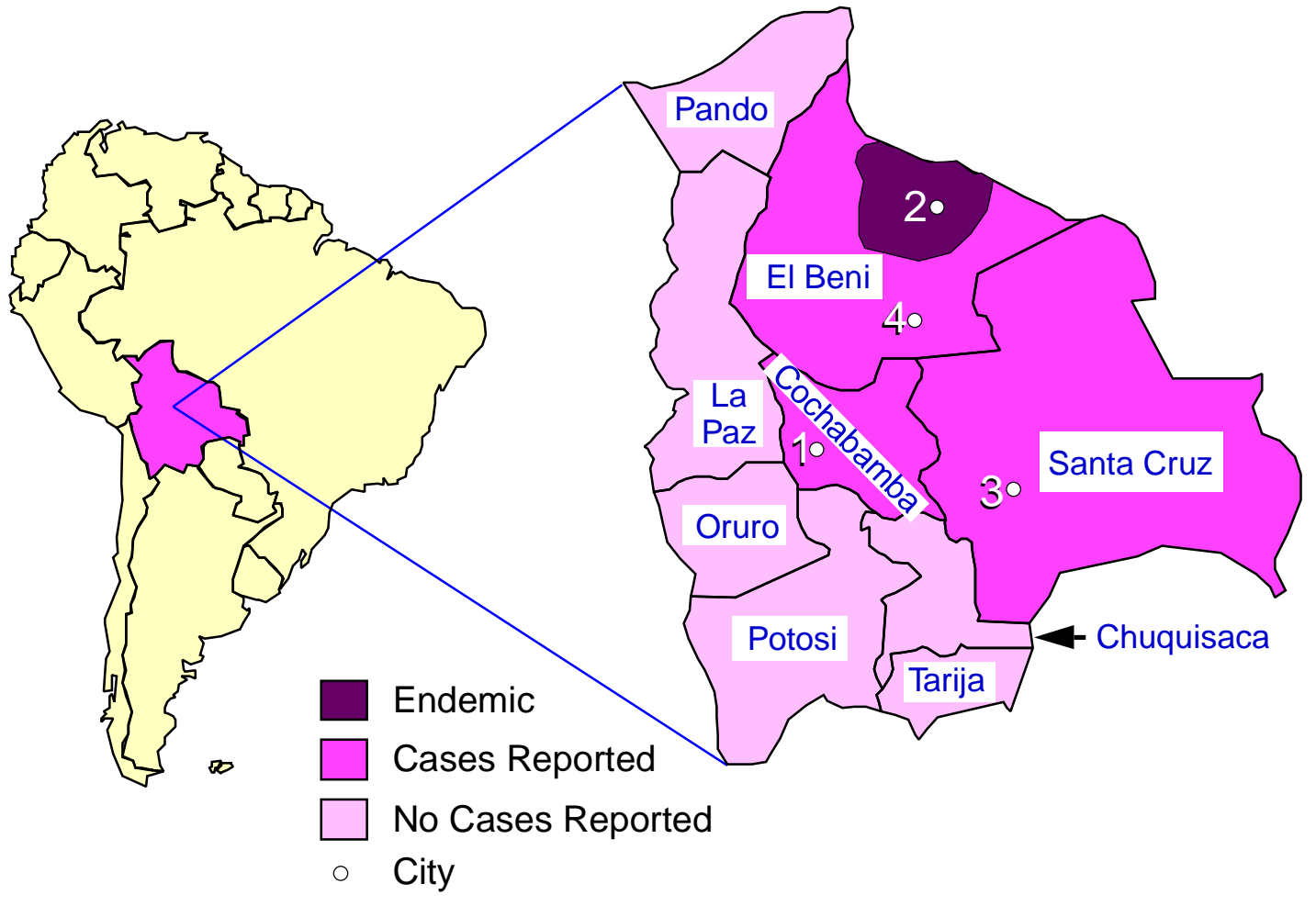

* 1) Cochabamba, 2) Magdalena, 3) Santa Cruz, and 4) Trinidad. 
Bolivian Hemorrhagic Fever - Continued

\section{Search for Other Cases}

Following identification of the familial cluster, three additional persons in Bolivia with suspected BHF were reported to the National Secretary of Health through provincial health departments. On August 18, a broken test tube in a centrifuge exposed a 37-year-old laboratory technician in Santa Cruz to aerosolized blood from one of the family members who died. On August 29, the technician developed an acute febrile illness with lower back pain, arthralgias, and mild conjunctivitis. On August 30, intravenous therapy with the antiviral compound ribavirin was initiated for a presumptive diagnosis of BHF. She had no hemorrhagic manifestations and recovered from her illness. Machupo antigen detection and virus isolation studies on serum obtained before initiation of ribavirin treatment were negative, as were $\lg G$ and $\lg M$ antibody ELISAs on serum specimens collected 3 months after onset.

On August 28, a 41-year-old man residing in Magdalena (with no known link to any infected persons) developed an illness that included fever, chills, and hip pain. On September 2, he was transferred to a hospital in Cochabamba, Bolivia, and died on September 5 following a fulminant hemorrhagic clinical course. Machupo virus was isolated and viral antigen was detected in the patient's serum.

On September 3, a 52-year-old agricultural worker from Poponas, El Beni Department, developed a febrile hemorrhagic illness; on September 11, he was admitted to a hospital in Trinidad, El Beni Department. On September 13, intravenous ribavirin therapy was initiated for a presumptive diagnosis of BHF, and the patient recovered. The diagnosis of BHF was confirmed by detection of viral antigen and virus isolation from the patient's serum.

Family members of these three persons with presumptive or confirmed BHF cases and health-care workers in contact with these persons were monitored for febrile illness. However, illness was not noted in these patient contacts.

\section{Rodent Investigation}

During August and September 1994, rodent trapping was conducted in areas of potential exposure for the affected family. During 1811 trap-nights, ${ }^{*} 84$ rodents were captured, including nine Calomys callosus. Testing for antibodies to Machupo virus was negative for each of the 84 rodents. Virus isolation studies on captured rodents are pending.

Reported by: M Villagra, MD, National Hemorrhagic Fever Program, National Secretary of Health, Ministry of Human Development; L Suarez, MD, Regional Health Secretary, El Beni Department; R Arce, MD, Magdalena Hospital, Magdalena, Province of Iténez, El Beni Department, Bolivia. MG Moreira, MD, Pan American Health Organization, La Paz, Bolivia. Div of Viral and Rickettsial Diseases, National Center for Infectious Diseases, CDC.

Editorial Note: BHF is a viral hemorrhagic fever known to be endemic only in Bolivia; first described in 1959, it caused outbreaks in small communities in eastern Bolivia throughout the 1960s (1). The etiologic agent, Machupo virus, is a member of the family Arenaviridae and is maintained in the rodent $C$. callosus, the natural reservoir (2). As with other arenaviruses, infection of the rodent host results in a persistent asymptomatic infection with shedding of virus in urine. Human infections are believed to occur following exposure to the virus in aerosolized rodent urine. A nosocomial

\footnotetext{
*The total number of traps set in 1 night multiplied by the total number of nights during which traps were set.
} 
Bolivian Hemorrhagic Fever - Continued

outbreak of BHF in Cochabamba in 1971 suggested that person-to-person transmission also may occur by airborne or parenteral routes (3).

Following an incubation period of 1-2 weeks, patients infected with Machupo virus may develop an influenza-like illness with fever, malaise, and fatigue followed by the onset of headache, dizziness, myalgias, and severe lower back pain. Prostration, abdominal pain, anorexia, tremors, and hemodynamic instability may be followed by hemorrhagic manifestations, including bleeding from the oral and nasal mucosa and the gastrointestinal, genitourinary, and bronchopulmonary tracts (4). BHF can be diagnosed by virus isolation from acute serum or tissue specimens or by virus antigen detection using an ELISA. Antibodies can be detected using plaque-reduction neutralization, indirect immunofluorescence, or ELISAs. Because of the risk for laboratoryacquired infections with this highly lethal agent, tests with potentially infectious material should be performed in a biosafety level 4 laboratory (5).

Treatment of BHF employs supportive measures. Although uncontrolled trials have used convalescent immune plasma from survivors of BHF, evaluation of the effectiveness of this therapy has been limited by the lack of plasmapheresis capability and availability of qualified donors. Ribavirin, a broad-spectrum antiviral agent, has been effective against human Lassa fever and several arenavirus diseases in animal models. Patients infected with Junin virus, a closely related arenavirus, also have received the drug (6), but there is no definitive evidence concerning efficacy.

From 1959 through 1962, Bolivian health officials reported 470 cases of BHF with 142 deaths (case-fatality rate: $30 \%$ ) (7). Until the cases described in this report, the last confirmed outbreak in Bolivia occurred in 1971 (3). The mode of transmission of BHF in the familial outbreak described in this report is unclear. Although no $C$. callosus were captured in the town of Magdalena, a low density of $C$. callosus was noted in rural areas around Magdalena where the index case had worked and traveled.

Previous trapping in Bolivia has shown fluctuations in population numbers and prevalence of infection among $C$. callosus, but the determining factors are not known $(2,8)$. Previous cases of BHF occurred following rodent invasion of households in towns and exposure during campestral activities, including sleeping in primitive shelters (9). Infection with Machupo virus among travelers returning to the United States has not been recognized.

\section{References}

1. Mackenzie RB, Beye HK, Valverde L, Garron H. Epidemic hemorrhagic fever in Bolivia. Am J Trop Med Hyg 1964;13:620-5.

2. Johnson KM, Kuns ML, Mackenzie RB, Webb PA, Yunker CE. Isolation of Machupo virus from wild rodent Calomys callosus. Am J Trop Med Hyg 1966;15:103-6.

3. Peters CJ, Kuehne RW, Mercado RR, Le Bow RH, Spertzel RO, Webb PA. Hemorrhagic fever in Cochabamba, Bolivia, 1971. Am J Epidemiol 1974;99:425-33.

4. Peters CJ. Arenaviruses. In: Belshe RB, ed. Textbook of human virology. Littleton, Massachusetts: PSG Publishing Company, Inc, 1984:513-45.

5. CDC. Biosafety in microbiological and biomedical laboratories. 3rd ed. Atlanta: US Department of Health and Human Services, Public Health Service, CDC, 1993:135-7; DHHS publication no. (CDC)93-8395.

6. Enria DA, Maiztegui Jl. Antiviral treatment of Argentine hemorrhagic fever. Antiviral Res 1994;23:23-31.

7. Hemorrhagic Fever Commission of Bolivia. Hemorrhagic fever in Bolivia [Spanish]. Bulletin of the Panamerican Health Office 1965;58:93-104.

8. Mercado R. Rodent control programmes in areas affected by Bolivian hemorrhagic fever. Bull World Health Organ 1975;52:691-6. 
Bolivian Hemorrhagic Fever - Continued

9. Stinebaugh BJ, Schloeder FX, Johnson KM, Mackenzie RB, Entwisle G, DeAlba E. Bolivian hemorrhagic fever. Am J Med 1966;40:217-29.

\section{Notice to Readers}

\section{Combined Issues of MMWR}

A December 30, 1994, issue of MMWR will not be published. Following that, the next issue will be Volume 43, Numbers 51 and 52, dated January 6, 1995, and will include the figure and tables on notifiable diseases and deaths for the weeks ending December 24 and December 31, 1994.

Notice to Readers

\section{Draft Recommendations for Prevention of Neonatal Group B Streptococcal Disease}

$\mathrm{CDC}$ is requesting public review of and comment on the draft document Prevention of Group B Streptococcal Disease: A Public Health Perspective. This document, which summarizes the literature and proposes prevention recommendations for neonatal group B streptococcal disease, has been published in the Federal Register*. Photocopies can be made at U.S. Government Depository Libraries and other public and academic libraries. Reprints and information for patients are available from CDC's Division of Bacterial and Mycotic Diseases, National Center for Infectious Diseases, telephone (404) 488-4202 or fax (404) 488-4632. Comments must be received in writing by February 15, 1995, at CDC, Attention: GBS Recommendations Review Committee, Mailstop C-09, 1600 Clifton Road, NE, Atlanta, GA 30333; fax (404) 639-3970.

*59 FR 64764-73. 


\section{CDC Professional and Technical Staff \\ Who Have Contributed to Editing, Producing, and Distributing Volume 43 of the MMWR Series}

\section{Epidemiology Program Office}

Deborah A. Adams

Roy C. Baron, M.D., M.P.H.

Phillip C. Bourque

Willard Cates, Jr., M.D., M.P.H.

Julie T. Creasy

Robert A. Fagan

Sandra L. Ford

Wanda T. Fortune

Karen L. Foster, M.A.

Richard A. Goodman, M.D., M.P.H.

Patsy A. Hall

Joy L. Herndon, M.S.

Suzanne M. Hewitt, M.P.A.

Morie M. Higgins

Peter M. Jenkins

David C. Johnson

Minnie L. Johnson

Denise Koo, M.D.

Carol M. Knowles

Arthur P. Liang, M.D., M.P.H.

William R. Mac Kenzie, M.D.

Nadine W. Martin

Patricia A. McGee

Ava W. Navin, M.A.

Barbara Panter-Connah

Darlene D. Rumph-Person

Teresa F. Rutledge

Myron G. Schultz, D.V.M., M.D.

Ruth W. Slade

Steven M. Teutsch, M.D., M.P.H.

Stephen B. Thacker, M.D., M.Sc.

T. Demetri Vacalis, Ph.D.

Scott F. Wetterhall, M.D., M.P.H.

Caran R. Wilbanks

Rachel J. Wilson

Lanette B. Wolcott

Information Resources Management Office

Jolene W. Altman

National Center for Infectious Diseases

Beverly J. Holland

Stephen M. Ostroff, M.D.

Office of the Deputy Director (HIV)

T. Stephen Jones, M.D., M.P.H.

Management Analysis and Services Office Carl T. Vining

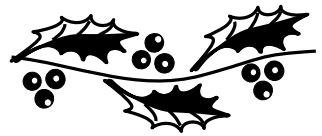


The Morbidity and Mortality Weekly Report (MMWR) Series is prepared by the Centers for Disease Control and Prevention (CDC) and is available on a paid subscription basis from the Superintendent of Documents, U.S. Government Printing Office, Washington, DC 20402; telephone (202) 783-3238.

The data in the weekly MMWR are provisional, based on weekly reports to CDC by state health departments. The reporting week concludes at close of business on Friday; compiled data on a national basis are officially released to the public on the succeeding Friday. Inquiries about the MMWR Series, including material to be considered for publication, should be directed to: Editor, MMWR Series, Mailstop C-08, Centers for Disease Control and Prevention, Atlanta, GA 30333; telephone (404) 332-4555.

All material in the MMWR Series is in the public domain and may be used and reprinted without special permission; citation as to source, however, is appreciated.

Director, Centers for Disease Control and Prevention David Satcher, M.D., Ph.D.

Deputy Director, Centers for Disease Control and Prevention Claire V. Broome, M.D.

Director, Epidemiology Program Office Stephen B. Thacker, M.D., M.Sc.

Editor, MMWR Series Richard A. Goodman, M.D., M.P.H.

Managing Editor, MMWR (weekly) Karen L. Foster, M.A.

Writers-Editors, MMWR (weekly) David C. Johnson Patricia A. McGee Darlene D. Rumph-Person Caran R. Wilbanks

U.S. Government Printing Office: 1995-533-178/05045 Region IV 